\title{
Institute for Research on Poverty
}

\section{Discussion Papers}

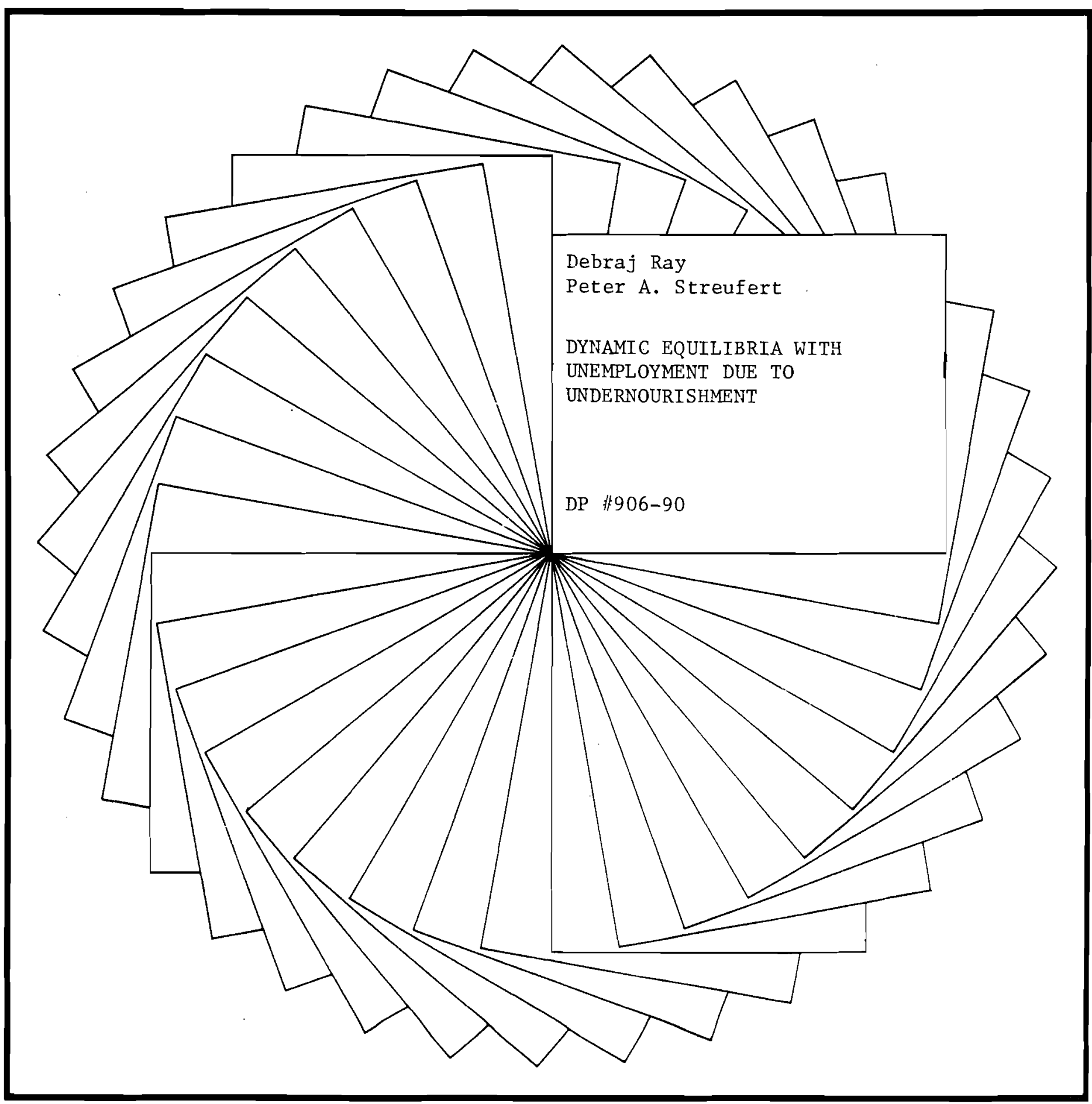


Institute for Research on Poverty

Discussion Paper no. 906-90

Dynamic Equilibria with Unemployment Due to Undernourishment

\author{
Debraj Ray \\ Indian Statistical Institute \\ Delhi Centre, India \\ Peter A. Streufert \\ Department of Economics \\ and \\ Institute for Research on Poverty \\ University of Wisconsin-Madison
}

February 1990

Many of our results were developed while Streufert visited the Indian Statistical Institute. Ray is grateful for financial support from the Warshow Endowment of Corne11 University, and Streufert thanks the Institute for Research on Poverty and the Graduate School, both at the University of Wisconsin-Madison. Any opinions expressed are those of the authors alone. 


\begin{abstract}
We provide characterization and stability results for the stationary equilibria of a competitive infinite-horizon model that incorporates the nutritional requirements of physical labor. We find that for many aggregate land stocks, there is a large continuum of stationary equilibrium unemployment rates. Since unemployment can be seen to stem from inequality in the initial distribution of land ownership, we suggest that certain land reforms can reduce unemployment.

In nontechnical terms, we assume that physical labor requires adequate nutrition, and that nutrition must be maintained over time by sufficient food consumption. We combine these key nutritional assumptions with standard economic assumptions: prices clear competitive markets; firms maximize profit while producing food from labor power and rented land; and persons maximize utility while consuming food, selling labor power and renting land to firms, and buying and selling land with one another. Typically we find that full employment is but one of many possibilities, and that higher rates of unemployment arise from greater inequality in the initial distribution of land. This unemployment arises in spite of competitive markets because the competitive wage alone is too low to provide adequate nutrition and thus persons without sufficient nonwage income from the rental of land cannot afford to work. Our work extends the literature by (1) modeling the dynamic nature of nutrition, (2) allowing persons to buy and sell land, and (3) providing initial observations about the ability of land reform to alleviate unemployment.
\end{abstract}


1. Introduction

We study the stationary equilibria of a competitive infinite-horizon model that incorporates the nutritional requirements of physical labor. Key parameters are the aggregate land stock and the initial distribution of land ownership.

For many aggregate land stocks, we find a large continuum of stationary equilibria. In certain equilibria, all persons are employed and well nourished. Yet in other equilibria from the same aggregate land stock, many persons are unemployed because they cannot meet the nutritional requirements of work. This result shows that unemployment and undernourishment can exist in spite of competitive dynamic markets and ample aggregate resources.

We also find that the root cause of unemployment and undernourishment is inequality in the initial distribution of land ownership. This suggests that land reform (in the sense of reducing the inequality of the initial land distribution) is an efficacious means of eradicating unemployment and undernourishment. Because increased employment raises aggregate output, this result directly contradicts the routinely cited equity-efficiency trade-off.

This paper grows out of the static efficiency-wage literature (Leibenstein (1957), Mirrlees (1975), Stiglitz (1976), Bliss and Stern (1978a, 1978b), Dasgupta and Ray (1986, 1987a)). Ours is the first competitive infinite-horizon model to incorporate the fact that work requires adequate nourishment (Guha (1987) and Gupta (1987) consider two-period models). In particular, we study each person's dynamic choice of body weight and land ownership in response to competitive prices. This rich model allows us to consider several issues that have not been previously addressed.

First, the nutritional requirement of work is inherently dynamic. The fundamental nonconvexity arises because labor requires a considerable body 
weight, and because most food intake is expended in maintaining body weight. The static efficiency-wage literature simply assumes body weight is constant, and then combines the above facts to derive a nonconvexity in the relationship between food intake and labor capacity (see Bliss and Stern (1978b), pp. 381-382). We do not assume constant body weight, but rather permit it to vary over time in response to a person's consumption and activity history. Thus we accommodate for the first time the dynamic nature of the fundamental nutritional assumptions.

Second, note that in Dasgupta and Ray's (1986, 1987a) static general equilibrium model, unemployment and undernourishment stem from inequality in the exogenous distribution of rental income from land ownership. We ask how that inequality in the distribution of land ownership will evolve over time. This question about agrarian structure is important in its own right. It is also important because Dasgupta and Ray's entire analysis would be crippled if the land distributions yielding unemployment could not be supported in stationary equilibrium. The answer is far from obvious. Unemployed persons, relative to employed persons, place a higher premium on increased land ownership because additional land not only earns the market rental rate, but also provides via this rental income the key to nourishment and employment. Since their return to land ownership is higher, it would seem that inequality would decrease over time. On the other hand, poor persons can sell land to buy food for the purpose of increasing their body weight and thereby securing employment. Wealthy persons can buy land without jeopardizing their ability to work. These observations would suggest that inequality will increase over time. It turns out that stationary equilibria with unemployment are among the many possibilities.

Third, we consider the stability of stationary equilibria to changes in the initial land distribution. We find that stationary equilibria are stable to small changes, and that large changes can greatly affect the unemployment rate. This is good news in the sense that our analysis is 
robust to small changes in the model's parameters. However, it is bad news in the sense that the real problems of unemployment and undernourishment can be robust to small land reforms. This stability analysis frames two important open questions. First, how drastic must a land reform be to jolt an economy out of a bad equilibrium? second, how can a land reform be implemented, not in the narrow sense of a change in the initial land distribution (as we consider here), but in the more important sense of an ongoing policy such as progressive capital taxation?

\section{Nutritional Assumptions}

\subsection{The Capacity Constraint}

Let $t \in\{1,2, \ldots\}$ be time (measured in days), let $m_{t} \in \mathbb{R}_{+}$be a person's body weight (measured in kilograms), and let $l_{t} \in \mathbb{R}_{+}$be a person's labor power (measured in tasks accomplished). We assume an s-shaped relationship between body weight and maximum labor capacity in accord with the experimental data of Keys (1950) (see Bliss and Stern (1978b), p. 381 , for details). This stylized relationship is shown in Figure 1, and it will be called the capacity constraint. The curve is not concave because a sizable portion of the human body is composed of internal organs and bones. (Implicitly we assume all persons are adults who are identical except for weight. In particular, all have the same height.)

Considerations of tractability force us to assume that $l_{t} \in\{0, \tilde{l}\}$, where $\tilde{l}>0$. Thus we assume that each day, each person is either unemployed or works at the fixed rate of $\tilde{l}$ units of labor power. (Clearly this assumption is unattractive because undernourished people actually do work for fewer hours and/or at less intensity than well-nourished people.\} Since $l_{t} \in\{0, \tilde{l}\}$ by assumption, we simplify our notation by introducing the binary variable $e_{t} \in(0,1)$ and the Identity $l_{t}=\tilde{i} e_{t}$. Thus $e_{t}=0$ denotes 


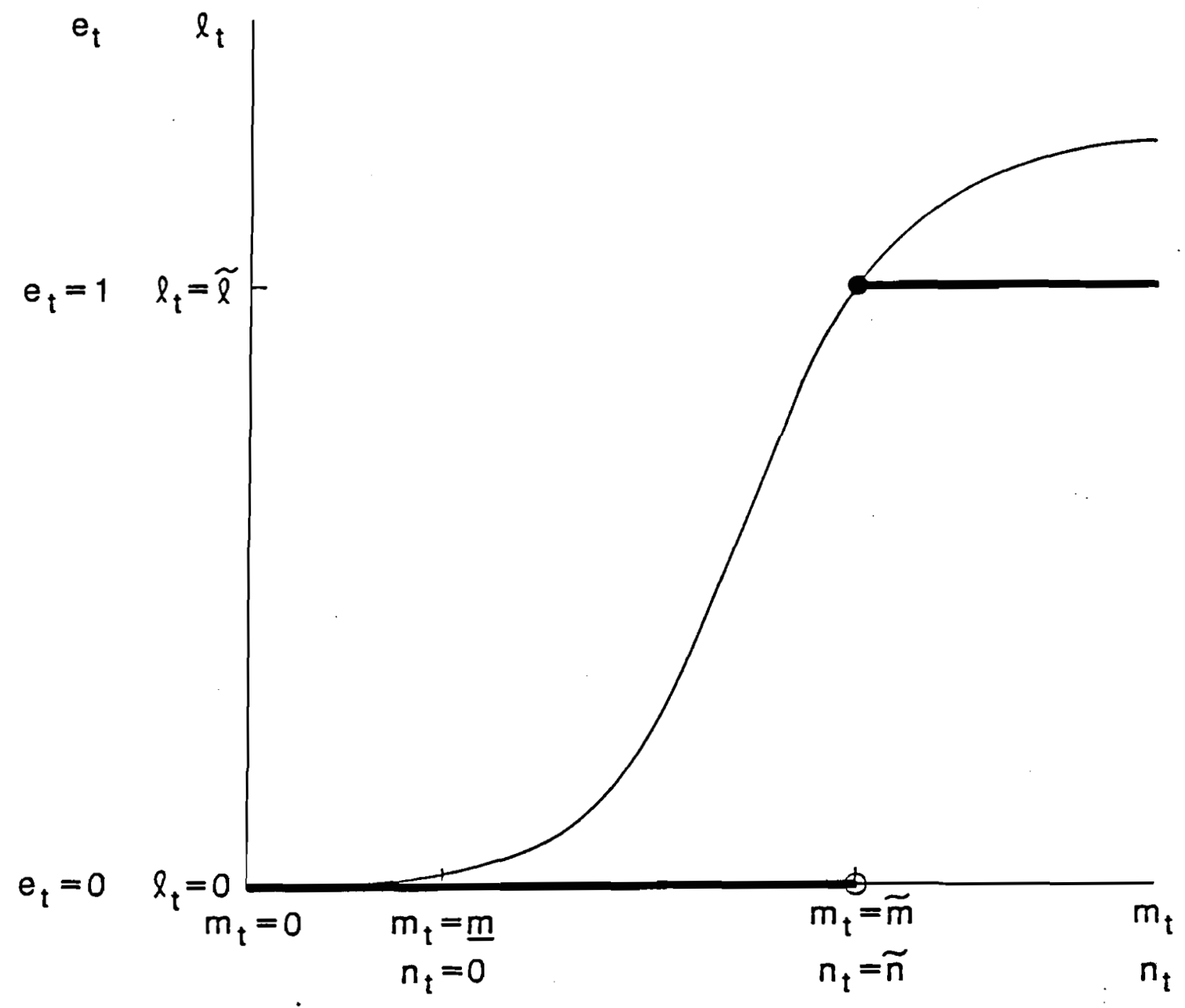

Figure 1: The true capacity constraint is shown by the light S-shaped curve in the variables $(m, l)$. The approximation (1) is shown by the dark step-function in the variables $(m, e)$. Equation (4) is shown by the dark step-function in the variables $(n, e)$. 
unemployment and $e_{t}=1$ denotes employment.

Due to the discreteness of $e_{t}$ ' we approximate the capacity constraint given by the curve in Figure 1 with the statement

$$
(\forall t \geq 1) e_{t}=1 \Rightarrow m_{t} \geq \tilde{m} \text {. }
$$

which corresponds to the step function in Figure 1 . Our economic model will be driven by the person-specific increasing returns to scale of (1). Note that this fundamental nonconvexity stems from the S-shaped capacity constraint and not from the discreteness of $e_{t}$.

\subsection{The Energy Balance Equation}

Let $f_{t} \in \mathbb{R}_{+}$be the day's consumption of food (measured in calories). We posit the following energy balance equation having income on the lefthand side and expenditures on the right-hand side:

$$
(\forall t \geq 1) E_{t}=\Phi_{1} m_{t-1}+\Phi_{2}^{l} t+\Phi_{3}\left(m_{t}-m_{t-1}\right)
$$

where $\Phi_{1}, \Phi_{2}$, and $\Phi_{3}$ are positive constants such that $\Phi_{3}>\Phi_{1}$.

The single left-hand term is food intake. For expositional ease, we neglect the distinction between calories consumed and calories absorbed into the bloodstream (Ray and streufert (1987) maintain this distinction and obtain virtually identical results.)

The first right-hand term gives the energy used to maintain the body under fasting conditions. It consists of the basal metabolic rate (which covers the energy needed to maintain tissues and to keep the heart and lungs moving) plus an additional 50\% of the basal metabolic rate (which covers minimal voluntary activity such as eating, digesting, and washing). This term is large. Bliss and stern ((1978b), pp. 373-374) cite FAO/WHO estimates of the daliy energy needs of the most active male farm worker: 
2600 calories are needed for maintenance compared to only 900 calories for work.

The second right-hand term gives the energy expended on work. First, let us reiterate that $l_{t}$ measures physical, not mental, work. In crude terms, the human body is a machine that converts one calorie of food energy into about $1 / 4$ calorie of work and wastes the remainder in heat (so that $\phi_{2}$ $=4$ if $l_{t}$ were measured in calories instead of tasks). Some research would suggest that the energy expended on work should increase with body weight (e.g.. Bliss and Stern $(1978 b)$, p. 373). This correlation seems to stem from the fact that heavier persons must carry more of their own weight. But, this proportional increase seems to be offset by the proportional increase in the size of the load that a heavier person can carry (see Dasgupta and Ray (1987b), p. 49). Thus we exclude body weight from this term.

The last right-hand term gives the energy stored by increasing body weight. One kilogram of fat stores about 4000 calories, while one kilogram of protein stores about 9000 calories. We disregard this distinction and take $\Phi_{3} \in(4000,9000)$. We also disregard the fact that some energy is lost in the storage and retrieval process. We assume $\phi_{3}>\phi_{1}$ since building new tissue costs more than mantaining old tissue for a day.

This equation is quite abstract, for it says that the human body stores all calories that remain after exogenously determined maintenance and labor expenditures. In fact, the maintenance and storage terms are endogenously determined by the human body. These two terms address many competing objectives (e.g., to resist disease, to store calories, and to build muscle and control weight), and different humans place different weights on these competing objectives depending upon theis personal and family histories. Dasgupta and Ray (1987b) and Srinivasan (1988) give further details from differing perspectives. In spite of its shortcomings, equation (2) does specify in a tractable fashion the indisputable facts 
upon which our model is built: labor requires energy, maintenance requires an even greater amount of energy, and insufficient caloric intake results in emaciation.

$$
\begin{gathered}
\text { Algebraic manipulation using the identity } l_{t}=\tilde{i} e_{t} \text { alters (2) to } \\
m_{t}=\beta m_{t-1}+g t-\alpha e_{t}
\end{gathered}
$$

where

$$
\begin{aligned}
\beta & =\left(\Phi_{3}-\Phi_{1}\right) / \Phi_{3}, \\
g_{t} & =\Phi_{t} / \Phi_{3}, \text { and } \\
\alpha & =\Phi_{2} \tilde{l} / \Phi_{3} .
\end{aligned}
$$

The exogenous constant $B$ lies in $(0,1)$ because $\phi_{1}$ is positive and strictly less than $\Phi_{3}$ by assumption. Thus $B$ may be regarded as a depreciation factor. The nonnegative scalar $g_{t}$ measures food consumption in new units (the new unit is equal to the number of calories that increases body weight by one kflogram). The exogenous constant a gives the decrease in body weight that would result from one day's employment while fasting.

The fundamental nonconvexity arises because labor requires a considerable body weight (equation (1)), and because most food intake is expended in maintaining body weight $\left(\phi_{1}\right.$ is large). The large magnitude of $\Phi_{1}$ now appears as the considerable difference between the depreciation factor $B$ and unity.

\subsection{Survival Probability}

Emaciation reduces one's resistance to disease and hence increases the probability of death. Thus survival probability is an increasing function of body weight lat least at the low levels of body weight which are relevant here). This observation is formally specified at the end of this section. It is economically relevant because a lower survival probability 
implies a smaller concern for consumption in the future (i.e., a higher rate of impatience).

Endogenous survival probability plausibly leads to endogenous population size. Yet considerations of tractability force us to assume a constant population size and to provide the following demographic interpretation: Each person who dies after period $t$ is replaced in period $t+1$ by a perfect clone, interpreted as the child of the deceased. In particular, this child inherits the body weight of its parent. This story accords crudely with the fact that in relatively undernourished dynasties (i.e., family lines), persons have shorter lifetimes and generations elapse more quickly.

This demographic interpretation is implausible if body weight can fall to zero. Thus we assume each person is endowed with $g \in(0,(1-\beta) \tilde{m})$ units of food each day and must consume it immediately (this minimal consumption is wholly exogenous to section $3^{\prime}$ s economic model). Define $m=(1-\beta)^{-1} \mathrm{~g} \epsilon$ $(0, \tilde{m})$. Equations $(1)$ and (3) imply that if $m_{0} \geq \underline{m}$, then $(\forall t \geq 1) m_{t} \geq \underline{m}$. The reader might regard $g$ as food gathered through gleaning or begging. This interpretation is flawed because all persons are assumed to get $g$ even if they are productively employed in the economic model. Yet the unemployment we exhibit in the economic model would only be exacerbated if persons were forced to abandon $g$ upon gaining employment.

$$
\text { In order to simplify notation, define } c_{t}=g_{t}-g \text { and } n_{t}=m_{t}-\underline{m} \text {. }
$$
Algebraic manipulation of (1) and (3) yields

$$
\begin{array}{ll}
(\forall t \geq 1) & e_{t}=1 \Rightarrow n_{t} \geq \tilde{n}, \text { and } \\
(\forall t \geq 1) & n_{t}=\beta n_{t-1}+c_{t}-\alpha e_{t^{\prime}}
\end{array}
$$

where $\tilde{n}=\tilde{m}-\underline{m}$. For expositional ease, we call $n_{t}$ nutrition rather than "weight in excess of $\underline{m}$. 
Finally, we formally specify survival probability by the function $P$ : $\mathbb{R}_{+} \rightarrow(0,1]$. Given current nutrition $n_{t}$, the value $P\left(n_{t}\right)$ gives the probability of being alive in the next period. We assume either exogenous survival, in which case $P$ is constant, or endogenous survival, in which case $P$ is continuous, strictly increasing below $\tilde{n}$, and constant above $\tilde{n}$. (The constancy of $P$ above $\tilde{n}$ will allow us to determine more easily the equilibrium interest rate.)

\section{The Economic Model}

\subsection{Land Ownership}

Let $k_{t}$ denote the amount of land owned by a dynasty at the end of period $t$. Let food be the numeraire, let $q$ be a stationary price for one day's labor power (i.e., for $\tilde{i}$ tasks accomplished), let $r$ be a stationary rental rate for one period's use of one unit of land, and let $p$ be a stationary price for one unit of land. Each dynasty is constrained to satisfy both

$$
\begin{gathered}
(\forall t \geq 1) k_{t}=k_{t-1}+\left(r k_{t-1}+q e_{t}-c_{t}\right) / p \\
\text { and }(\forall t \geq 1) k_{t} \geq 0 .
\end{gathered}
$$

Equation (6) states that income in excess of consumption is used to purchase land (rental and wage income are the only conceivable sources of income since we will postulate constant returns to scale and no other inputs). Although land may be sold, (Z) stipulates that a dynasty may not own a negative amount of land (i.e., go into debt). Equation ( 7 ) is reasonable because of the incentive problems inherent in borrowing against future income, and because debt cannot be inherited by future generations. Debt is further discussed in section 4.4. 


\subsection{Preferences}

The objective function of each dynasty is

$$
\sum_{t=1}^{\infty} \delta^{t-1}\left[\pi_{s=1}^{t-1} P\left(n_{s}\right)\right] G\left(c_{t}\right) .
$$

where $\delta \in(0,1)$ is a discount factor, $P: \mathbb{R}_{+} \rightarrow(0,1]$ is the survival probability function defined above, and $G: \mathbb{R}_{+} \overrightarrow{\mathbb{R}_{+}}$is a single-period utility function. Assume that $G$ is strictly increasing, strictly concave, and continuously differentiable over $\mathbb{R}_{++}$. Also assume that there is some constant $\gamma<1$ such that for sufficiently large $c, G(c) \leq c^{\gamma}$.

The dynasty seeks to maximize (8) subject to the constraints $(4)-(7)$ given prices $(p, q, r)$ and its initial endowment of land and nutrition $\left(k_{0}, n_{0}\right)$. A solution to this problem is a sequence $\left\langle\left(c_{t}, e_{t}, k_{t}, n_{t}\right)\right\rangle_{t=1}^{\infty}$. Such a solution is stationary if the subscript $t$ can be dropped.

The dynasty's maximization problem has a natural interpretation: every person in the dynasty maximizes expected utility. (The utility function of the person living in period $u$ is $\left.\Sigma_{t=u}^{\infty} \delta^{t-u}\left[\pi_{s=u}^{t-1} P\left(n_{s}\right)\right] G\left(c_{t}\right).\right)$ This interpretation rests upon the simplified demography of section 2.3 , no intergenerational altruism, zero single-period utility after death, and the assumption that each child inherits the land and nutrition of 1 ts deceased parent.

\subsection{Production}

In each period, food is produced from land and labor power through a production function $F: \mathbb{R}_{+}^{2} \rightarrow \mathbb{R}_{+}$. Assume that $F$ is concave and constantreturns-to-scale, and that $F$ is strictly increasing and continuously differentiable over $\mathbb{R}_{++}^{2}$. Also assume that $\lim m_{K \rightarrow \infty} F(K, 1)=+\infty$, and that $F$ satisfies the Inada endpoint conditions: $F(0,1)=F(1,0)=0$ and $\lim _{K \rightarrow 0}$ $F_{K}(K, 1)=\lim _{E \rightarrow 0} F_{E}(1, E)=+\infty$. The reader may interpret $F$ either as a 
production technology for one giant competitive firm, or as a technology available to all persons in the economy. Due to constant returns to scale, both are equally consistent with our analysis.

\subsection{Stationary Equilibrium}

Let $\mathrm{K}>0$ be the aggregate land stock in the economy. Let there be a continuum of dynasties, indexed by $f$ and distributed uniformly on $[0,1]$. A stationary equilibrium consists of the following (time-stationary) objects: prices $(p, q, r) \in \mathbb{R}_{++}^{3}$, a consumption distribution $c:[0,1] \rightarrow \mathbb{R}_{+}$, an employment distribution $\mathrm{e}:[0,1] \rightarrow\{0,1\}$, a land distribution $k:[0,1] \rightarrow \mathbb{R}_{+^{\prime}}$ and a nutrition distribution $\mathrm{n}:[0,1] \rightarrow \mathbb{R}_{+}$. This collection of prices and distributions is a stationary equillbrium if it satisfies the following four properties (implicitly, $c, e$, and $k$ must be Lebesque( $\mu$ )-measurable).

(a) For every $j \in[0,1],(c(j), e(j), k(j), n(j))$ is a stationary solution to the maximization problem (4)-(8) given prices $(p, q, r)$ and initial stocks $(k(j), n(j))$.

(b) The aggregate land stock $\mathrm{K}$ is distributed by $\mathrm{k}: \mathrm{J} \mathrm{k} d \mu=\mathrm{K}$.

(c) The input markets clear given competitive behavior by the producer(s): $(K, J$ e d $\mu)$ maximizes $F\left(K^{\prime}, E^{\prime}\right)-r K^{\prime}-q E^{\prime}$ over $\left(K^{\prime}, E^{\prime}\right) \in \mathbb{R}_{+}^{2}$.

(d) The food market clears: $\int$ c d $\mu=F\left(K, \int\right.$ e du).

\section{Characterization}

\subsection{Characterization Theorem}

Define the employment rate $E$ by $E=\int$ e du. We will characterize the various employment rates $E$ (and the unemployment rates $1-E$ ) that can prevail as the aggregate land stock $\mathrm{K}$ is parametrically varied. Define the viable threshold $\underline{K}$ by 


$$
F(\underline{K}, 1)=(1-\beta) \tilde{n}+\alpha,
$$

and define the full-employment threshold $\overline{\mathrm{K}}$ by

$$
F_{E}(\bar{K}, 1)=\tilde{n}+\alpha
$$

( $\underline{\mathrm{K}}$ and $\overline{\mathrm{K}}$ exist because of the Inada conditions and the assumption that $\left.\lim _{K \rightarrow \infty} F(K, I)=+\infty\right)$. Note that $\vec{K}$ must lie substantially above $\underline{K}$. The reason for our nomenclature will soon become clear.

Characterization Theorem: For any land stock $K$, there is a stationary equilibrium with employment rate $E$ (and unemployment rate $1-E$ ) if and only if both of the following conditions are met:

$$
\begin{aligned}
& K / E \geq \underline{K} \text { and } \\
& K / E \geq \bar{K} \Rightarrow E=1 .
\end{aligned}
$$

(The Appendix contains a constructive proof.)

See Figure 2. Note that ald (K,E) pairs having the same land/employment ratio $\mathrm{K} / \mathrm{E}$ form a line passing through the origin. For instance, if $\mathrm{K}$ $<\overline{\mathrm{K}}$, the Characterization Theorem states that the equilibrium $\mathrm{K} / \mathrm{E}$ ratio can lie anywhere between $\underline{K}$ and $\overline{\mathrm{K}}$. The following three subsections provide intuition for the theorem and argue that a higher $\mathrm{K} / \mathrm{E}$ (1.e., a higher unemployment rate for a given $K$ ) stems from greater inequality in the initial distribution of land.

\subsection{Beyond the Full-Employment Threshold $\overline{\mathrm{K}}$}

Equation (10) states that unemployment is inconsistent with a land/employment ratio $\mathrm{K} / \mathrm{E} \geq \overrightarrow{\mathrm{K}}$. The intuition is straightforward: By the definition of $\bar{K}, K / E \geq \bar{K}$ implies that the equilibrium wage is at least $\tilde{n}+\alpha$, in which case even a landless and emaciated person could afford to become 


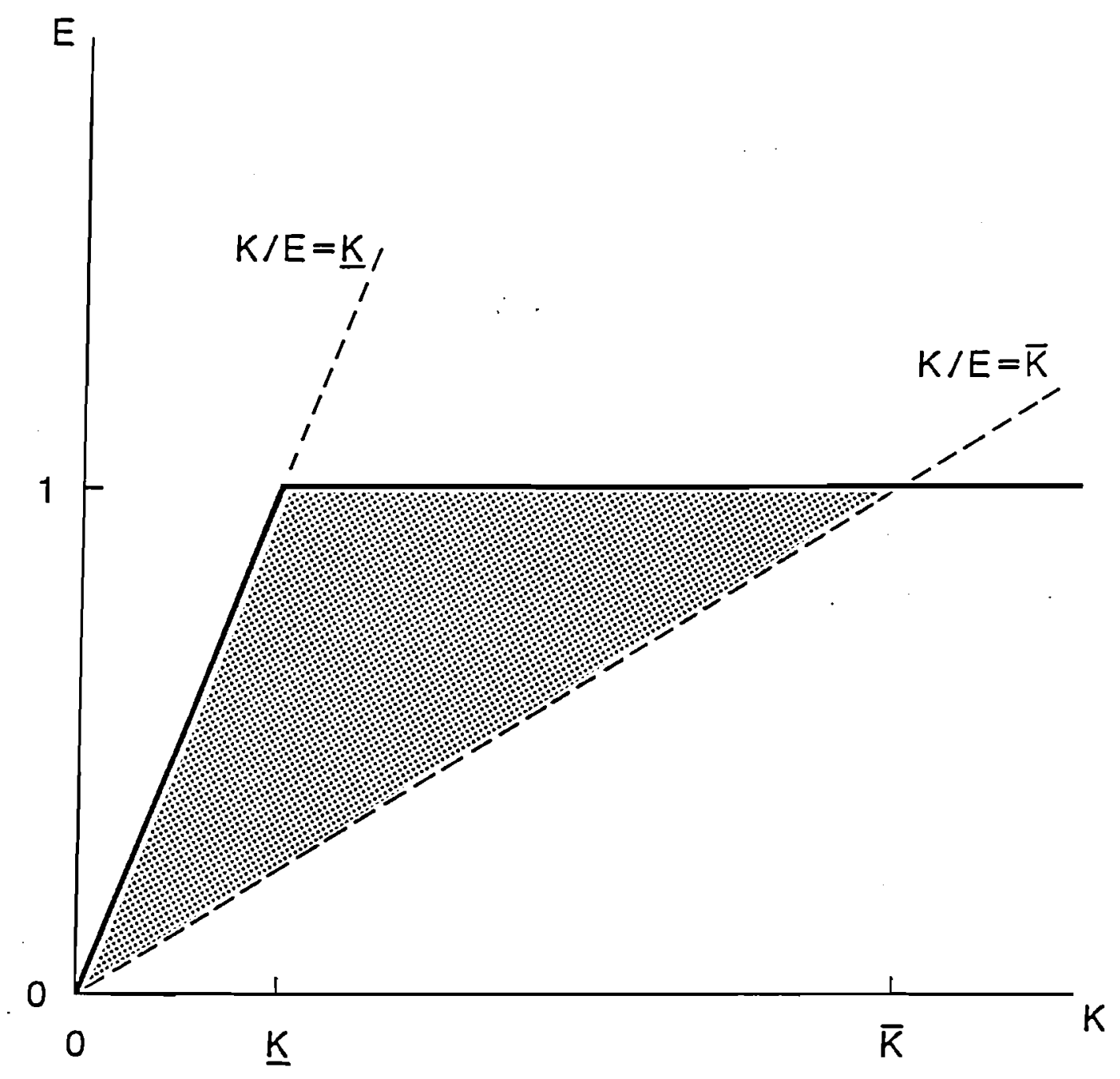

Figure 2: Given aggregate land stock $\mathrm{X}$, there is a stationary equilibrium with employment rate $E$ (and unemployment rate $1-E$ ) if and only if the pair (X,E) is on the solid line or within the shaded triangle. 
adequately nourished and to work. If $K \geq \vec{K}$, then $K / E$ must exceed $\bar{K}$, and consequently, full employment is inevitable. Thus $\overline{\mathrm{K}}$ can be thought of as a threshold beyond which "trickle-down" effects remove the "poverty trap" analyzed in this paper.

\subsection{Between the Viable and Full-Employment Thresholds}

Unemployment is possible when $\mathrm{K}$ lies below the full-employment threshold $\vec{k}$. For intuition, consider any employment rate $E<1$ such that the equilibrium wage $F_{E}(K / E, 1)$ lies below $F_{E}(\bar{K}, 1)=\tilde{n}+\alpha$ (such an E exists by $K<\vec{K}$ and the definition of $\bar{K}$ ). Endow each unemployed dynasty with zero land and zero nutrition. Since the wage lies below $\tilde{n}+\alpha$, these luckless dynasties have but one feasible path satisfying (4)-(7), and it entails perpetual unemployment and zero consumption. (Stabilfty Theorems 1 and 2 show that a similar argument holds when the luckless dynasties are endowed with small positive stocks.) Endow each employed dynasty with $\mathrm{K} / \mathrm{E}$ units of land and at least $\tilde{n}$ units of nutrition. These fortunate dynasties will consume $r(K / E)+g=F(K / E, 1)$ in each period, and this covers their nutritional requirements of work because $F(K / E, 1) \geq F(K, 1) \geq F(\underline{K}, 1)=(1-\beta) \tilde{n}+\alpha$ by $K \geq \underline{K}$ and the definition of $\underline{K}$. Notice that unemployment and undernourishment are generated by inequality in the initial distribution. Extreme inequality is needed to support equilibria having employment rates near the lower bound imposed by $(10)$.

Full employment is also possible when $K$ lies above the viable threshold $\underline{K}$. Simply endow each dynasty with land $K$ and nutrition $\tilde{n}$. Everyone will consume $r K+q=F(K, 1)$ in each perlod, and this covers the nutritional requirements of work because $F(K, 1) \geq F(\underline{K}, 1)=(1-\beta) \tilde{n}+\alpha$ by $K \geq \underline{K}$ and the defintion of $\underline{K}$. Thus egalitarianism implies full employment when $\mathrm{K} \geq \underline{\mathrm{K}}$. 
The full-employment threshold $\overline{\mathrm{K}}$ lies far above the viable threshold $\underline{\mathrm{K}}$ : $\vec{K}$ is such that the wage alone suffices to renew the labor capability of an emaciated person, while $\underline{K}$ is such that total output per worker can maintain a worker's nutrition. This large region between $\underline{K}$ and $\bar{K}$ is of greatest interest, for here, there are competitive equilibria attaining full employment and adequate nutrition, and also competitive equilibria that do not come close to achieving this standard. We feel that this zone is a caricature-but a useful one-of many developing countries, where resources are adequate but unemployment and undernutrition nevertheless prevail. our analysis reveals that both good and bad outcomes are consistent with competitive markets, and that the initial distribution of land is critical.

\subsection{Below the Viable Threshold $\underline{K}$}

When $K<\underline{K}$, condition (10) continues to place a lower bound on the employment rate. In addition, condition (9) now imposes an upper bound that is strictly less than unity. This upper bound on employment (1.e., lower bound on unemployment) is purely technological. To be specific, let us say that $E$ is viable from $K$ if there exist stationary consumption, employment, and nutrition distributions $(c, e, n)$ that enable the employment rate $E$ in accord with $(4),(5)$, and $\int c d \mu \leq F(K, E)$. Lemma 1 (Appendix) shows that $E$ is viable from $K$ if and only if $K / E \geq \underline{K}$. Thus condition (9) shows that any viable employment rate can be supported in a stationary equilibrium (subject of course to the lower bound of (10)).

\subsection{Efficiency}

One measure of efficiency is production efficiency, as measured by aggregate food production. Since aggregate production increases with employment (the domain of $F$ is simply the nonnegative quadrant in Figure 2), equilibria with unemployment are plainly production inefficient 
whenever $K \in[\underline{K}, \bar{K})$. In this sense, initial inequality can lead to permanent inefficiency in the aggregate.

Pareto efficiency is another criterion. Due to the incomplete financial markets imposed by (7), stationary equilibria may not be Pareto efficient. In particular, we argue below that some equilibria in the lower half of Figure 2's triangle are Pareto inefficient, while all equilibria in the upper half of Figure 2's triangle are Pareto efficlent.

Define $K^{\circ}$ by $F_{E}\left(K^{0}, 1\right)=(1-\beta) \tilde{n}+\alpha$. Since $K<K^{0}<\bar{K}$, the equation $K / E=K^{\circ}$ divides Figure $2^{\prime} s$ triangle. If $K / E>K^{\circ}$, then the stationary equilibrium wage exceeds the stationary nutritional requirements of employment. Some of this surplus could be used to repay a loan that enabled an emaciated worker to accumulate nutrition $\tilde{\mathrm{n}}$. Thus such stationary equilibria might be upset by complete financial markets and might fail to be Pareto efficient. On the other hand, if $\mathrm{K} / \mathrm{E}<\mathrm{K}^{\circ}$, then the stationary equilibrium wage is insufficient to cover the stationary nutritional requirements of employment. Thus a worker endowed with no land and nutrition $\tilde{\mathrm{n}}$ could not afford to work, and hence could not repay a loan. Therefore we conjecture that these stationary equilibria will not be upset by complete financial markets, and hence will prove to be Pareto efficient.

\section{Stability}

We divide our discussion of stability into two parts. First, we consider "individual stability", that is, we take stationary equilibrium prices as given and study a dynasty (of measure zero) whose initial stocks of land and nutrition entail a nonstationary optimum. We ask whether or not the optimum converges over time to a stationary optimum. Second, we make some remarks on "system stability", that is, we consider initial distributions of land and nutrition that are not stationary equilibrium distributions, and we ask how those distributions evolve over time in a 
nonstationary dynamic general equilibrium. In this case, prices can vary over time. Individual stability is addressed formally in section 5.1 , while the much more difficult issue of system stability is addressed verbally in section 5.2 .

\subsection{Individual Stability}

Land $k$ and nutrition $n$ may be usefully regarded as a dynasty's two stock variables. Given prices $(p, q, r)$, a land-nutrition pair $(k, n)$ is optimal if there exists $(c, e)$ such that $(c, e, k, n)$ is a stationary solution to the optimization problem (4)-(8). The constraints (4)-(7) imply that every optimal land-nutrition pair without employment $(e=0)$ lies within

$$
\underline{s}=\left\{(k, n) \mid n=r k(1-\beta)^{-1}\right\}
$$

Similarly, the constraints $(4)-(7)$ imply that every optimal land-nutrition pair with employment $(e=1)$ lies within

$$
\bar{s}=\left\{(k, n) \mid n=(r k+q-\alpha)(1-\beta)^{-1} \text { and } n \geq \tilde{n}\right\}
$$

For future reference, we define the point $(\bar{k}, \bar{n})$ to be the unique element of $\vec{s}$ that satisfies either $n=\tilde{n}$ or $k=0$.

The curves $\underline{S}$ and $\bar{S}$ shift with the factor prices $(r, q)$, which (In equilibrium) depend solely on the land/employment ratio $\mathrm{K} / \mathrm{E}$. Figures 3 and 4 show $\underline{S}$ and $\bar{S}$ when $\bar{k}>0$, and Figure 5 shows $\underline{S}$ and $\bar{s}$ when $\bar{k}=0$ (only the lower portion of $S$ is shown since the rest is irrelevant). It may be instructive to note that $\bar{k}>0$ if and only if $K / E<K^{\circ}$. Section 4 of Ray and Streufert (1987) characterizes all stationary equilibrium land distributions in terms of such figures.

A set $Q$ consisting of optimal (stationary) land-nutrition pairs is a global attractor if, at each inftial $\left(k_{0}, n_{0}\right) \in \mathbb{R}_{+}^{2}$, an optimum to (4)-(8) exists and every optimum to $(4)-(8)$ satisfies $\lim _{t \rightarrow \infty}\left(k_{t}, n_{t}\right) \in Q$. Such a 


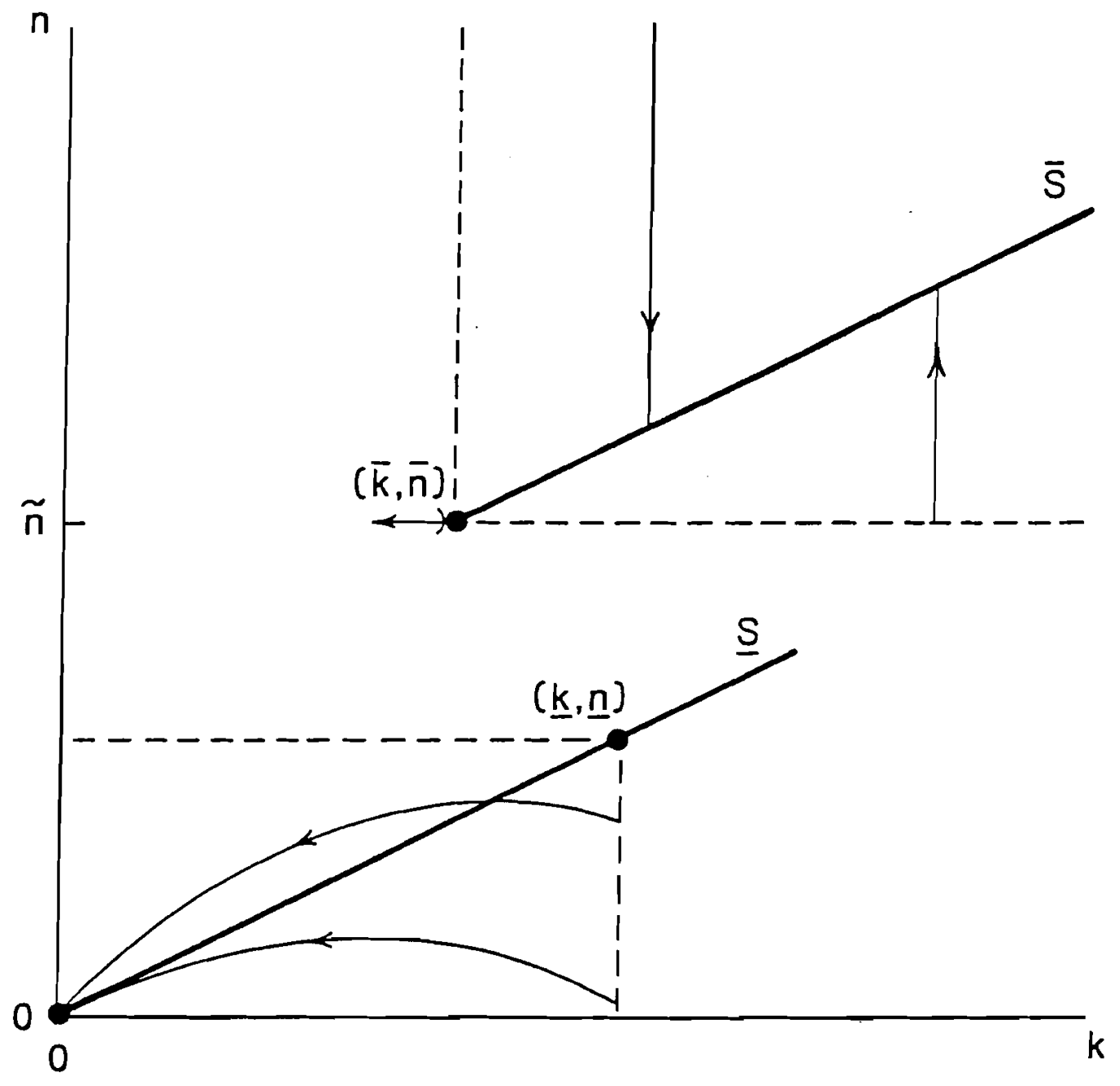

Figure 3: Stability Theorem 1 for $K<\bar{K}$ and endogenous survival. 


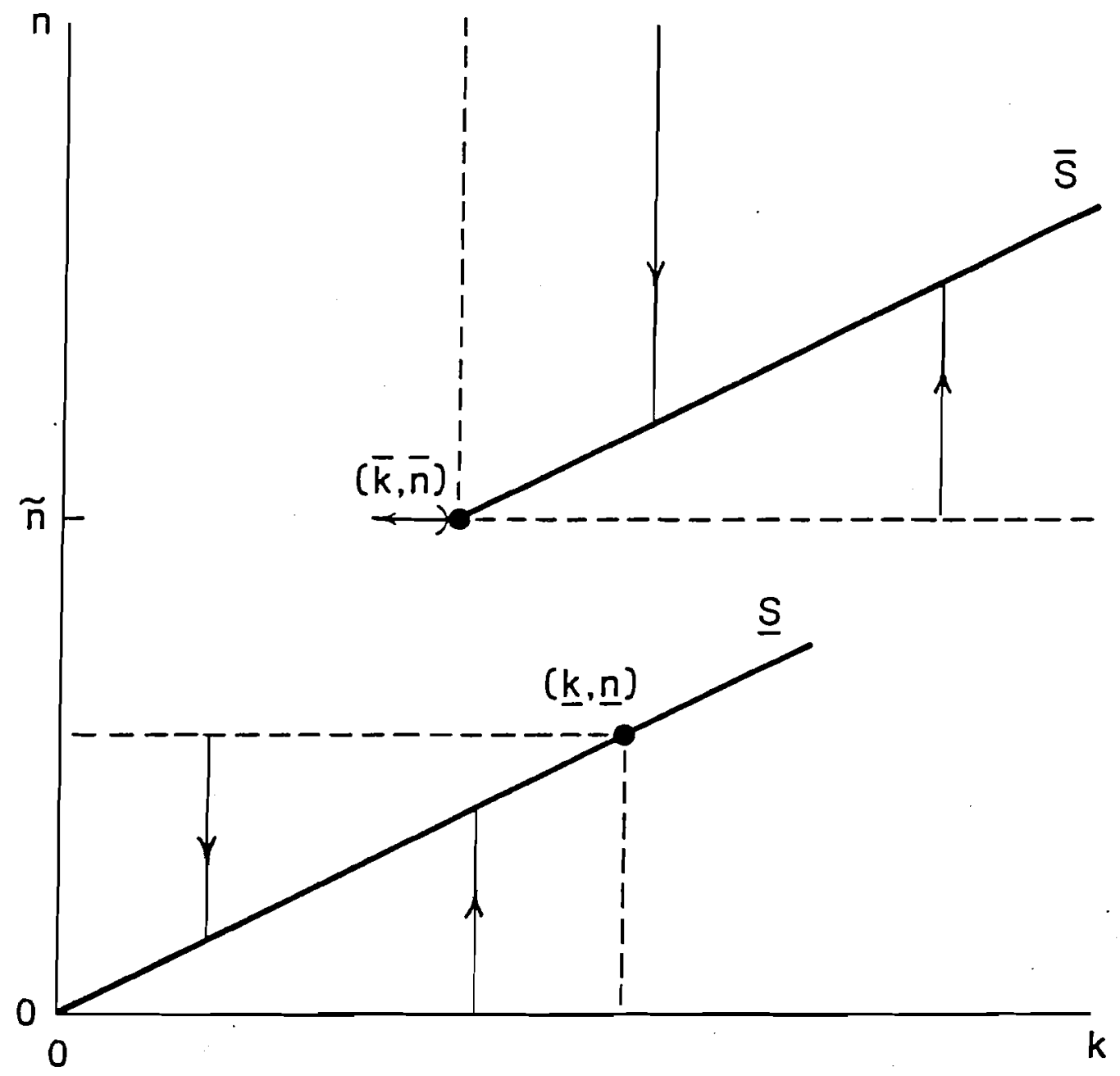

Figure 4: Stability Theorem 2 for $\mathrm{K}<\overline{\mathrm{K}}$ and exogenous survival. 
set $Q$ is a local attractor if there is an open set $B$ containing $Q$ such that if $\left(k_{0}, n_{0}\right) \in B$, then an optimum to $(4)-(8)$ exists and every optinum to (4)(8) satisfies $\lim _{t \rightarrow \infty}\left(k_{t}, n_{t}\right) \in Q$. An attractor $Q$ is monotonic if the optima converging to $Q$ also satisfy either $(\forall t \geq 1) k_{t} \geq k_{t-1}$ or $(\forall t \geq 1) k_{t} \leq k_{t-1}$.

Henceforth we assume that $(1+r / p)^{-1}=\delta P(\tilde{n})$. In other words, we assume that the equilibrium discount factor $(1+r / p)^{-1}$ is determined by $\delta P(\tilde{n})$, which is the subjective discount factor of all employed dynasties. This assumption is satisfied by all equilibria constructed in the proof of the Characterization Theorem. In addition, Lemma 4 (Appendix) shows that this assumption is satisfied by all equilibria in which some dynasty's $(k, n)$ lies on $\bar{s}$ above $(\bar{k}, \bar{n})$. Since all employed dynasties must be on $\bar{s}$, this lemma covers all equilibria except those singular cases in which all employed dynasties sit exactly at $(\bar{k}, \bar{n})$.

Stability Theorem 1: Assume $K<\overline{\mathrm{K}}$ and endogenous survival. Take any stationary equilibrium prices such that $(1+r / p)^{-1}=\delta P(\tilde{n})$. Then $\bar{S} \sim\{(\bar{k}, \vec{n})\}$ and $\{(0,0))$ are monotonic local attractors. However, $\vec{s}$ is not necessarily a monotonic local attractor even though $(\vec{k}, \vec{n})$ is optimal. (See Figure 3 , and Lemmas 5, 6, 8, and 10 in the Appendix.)

Stability Theorem 2 : Assume $\mathrm{K}<\overline{\mathrm{K}}$, exogenous survival, and lim $\mathrm{c} \rightarrow 0$ $G^{\prime}(c)=+\infty$. Take any stationary equilibrium prices such that $(1+r / p)^{-1}=$ $\delta P(\tilde{n})$. Then $\bar{S} \sim\{(\bar{k}, \bar{n})\}$ is a monotonic local attractor, and there is a landnutrition pair $(\underline{k}, \underline{n}) \in \underline{S} \sim\{(0,0)\}$ such that $\underline{S}\{(k, n) \mid(k, n)<<(\underline{k}, \underline{n})\}$ is a monotonic local attractor. However, $\overline{\mathrm{S}}$ is not necessarily a monotonic local attractor even though $(\bar{k}, \bar{n})$ is optimal. (See Figure 4, and Lemmas 5, 6, 9 , and 10 in the Appendix.)

Essentially, this pair of stability theorems says that all the stationary optima of (4)-(8) are locally stable, with the exception of $(\bar{k}, \bar{n})$. It is not surprising that optima in $\bar{s}-\{(\bar{k}, \bar{n})\}$ are stable, for near 
this set, the nutritional requirement of employment is not binding. Thus, the strict concavity of $G$ implies that dynasties consume the same amount in every period: $r k_{0}+q$.

It is important that the low stationary optima on $\underline{\mathbf{S}}$ are locally stable, for if these optima were locally unstable, our entire analysis could be crippled by arguing that any slight increment to the assets of the poor would permit them to climb out of poverty. The key to this result is the observation that there is some neighborhood of the origin from which persons would not ever seek employment by accumulating the necessary land and nutrition. Because land generates rental income and nutrition depreciates, a person seeking to gain employment would first accumulate land rather than nutrition. By buying more land with the rental income, the person's land stock could accumulate at a factor of $(1+r / p)$. Given endogenous survival (Stability Theorem 1), this accumulation is unattractive because the survival probability of undernourished persons falls below $P(\tilde{n})$, and hence their subjective discount factor falls below $\delta P(\tilde{\Omega})=$ $(1+r / p)^{-1}$. Given exogenous survival and sufficient desire for consumption smoothing (Stability Theorem 2), accumulation is undesirable because the desire for consumption smoothing makes low consumption during the accumulation process very onerous.

Although it was surprising to us that $(\vec{k}, \bar{n})$ is unstable, the result is intuitive. If $n_{0}=\tilde{n}$ and $k_{0}$ is just a little less than $\bar{k}>0$, the person could work for many periods while maintaining his land close to $\bar{k}$. In each perlod, he would sell a tiny bit of land to make up for the slight deficiency in rental income. In this case, $\left\langle k_{t}\right\rangle_{t=1}^{\infty}$ is clearly moving away from $\bar{k}$. The alternative to this scenario is to forego employment in the first period and to accumulate. This second option entails a large loss in consumption during the first perlod, and it is thus less attractive than the first option for persons whose $k_{0}$ is very close to $\bar{k}$. The instability 


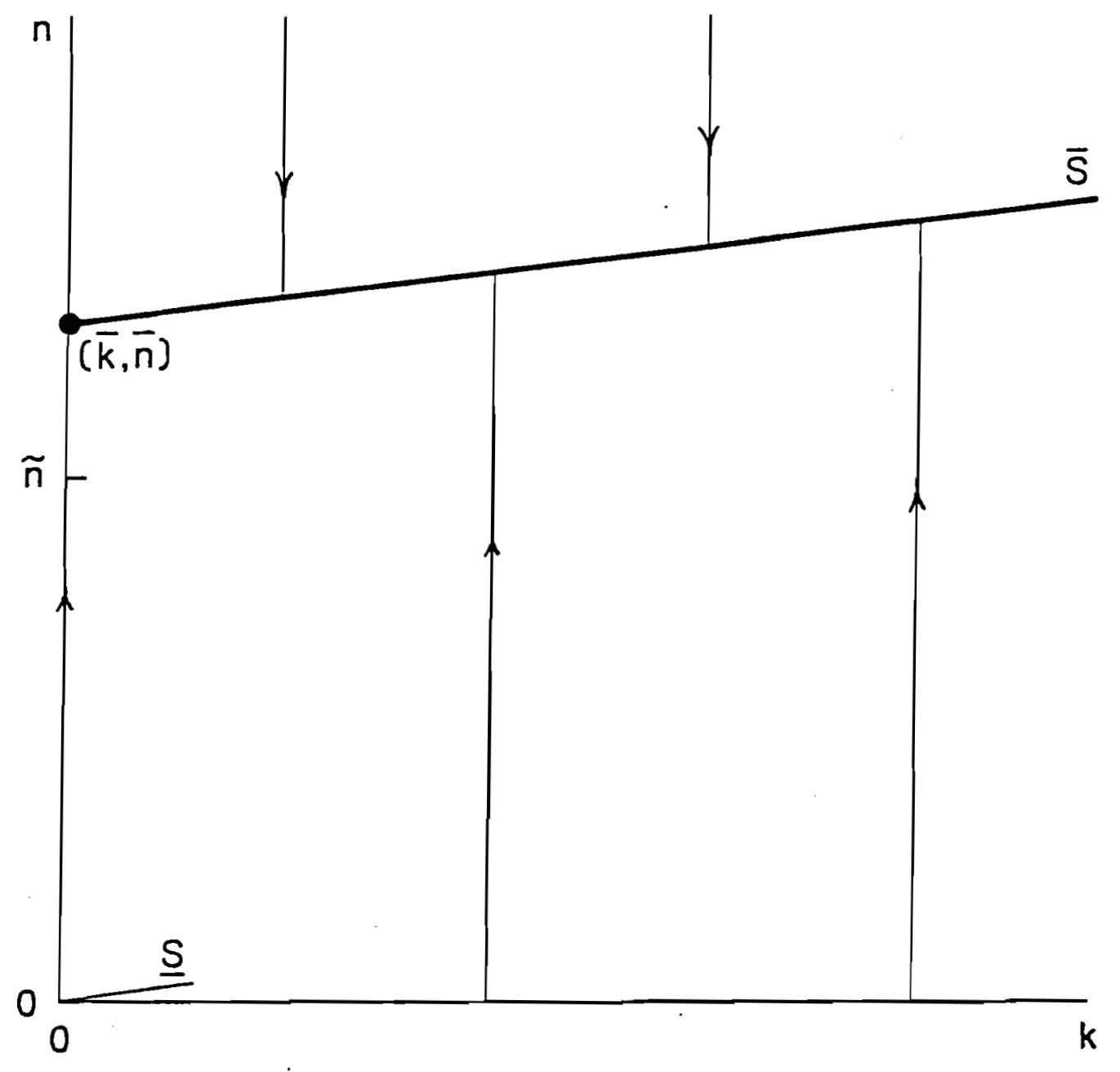

Figure 5: Stability Theorem 3 for $K \geq \bar{K}$. 
of $(\bar{k}, \bar{n})$ illustrates that it is technically difficult to solve the dynasty's maximization problem when $\left(k_{0}, n_{0}\right)$ lies between $(\underline{k}, \underline{n})$ and $(\bar{k}, \bar{n})$. Finally, if $K \geq \bar{K}$, the nutritional requirement for employment is never binding and every dynasty consumes $r k_{0}+q$ in every time period. Consequently, the following theorem is straightforward.

Stability Theorem 3 : Assume $K \geq \bar{K}$. Take any stationary equilibrium prices such that $(1+r / p)^{-1}=\delta P(\tilde{n})$. Then $\bar{S}$ is a monotonic global attractor. (See Figure 5, and Lemma 11 in the Appendix.)

\subsection{System Stability}

By "land reform" we mean a change in the inftial land distribution (1.e., an exercise in comparative dynamics). This entails issues of system stability because arbitrary changes in the initial land distribution will generally entail nonstationary equilibrium prices.

Let the status quo be a stationary equilibrium in which $K \in[\underline{K}, \bar{K})$, $E<1$, all unemployed are landless, and all the employed have $\mathrm{K} / \mathrm{E}$ units of land each (such equilibria are constructed in the Characterization Theorem's proof). After land reform, let the new land distribution be such that each originally unemployed dynasty has $\theta \mathrm{K}$ units of land, and each originally employed dynasty has $(1-\theta) K / E+\theta K$ units of land, where $\theta \epsilon$ $[0,1]$. Note that $\theta=0$ yields the status quo, and $\theta=1$ yields egalitarianism.

First assume exogenous survival. Stability Theorem 2 (Figure 4) indicates that sufficiently small land reforms ( $\theta$ near zero) will not have any effect on unemployment. Rather, the stationary equilibrium prices will be unaffected, the new land distribution will not change over time, and the rental income will have been permanently redistributed to the benefit of the unemployed. On the other hand, section 4.3 (second paragraph) suggests that sufficiently large land reform ( $\theta$ near one) will eradicate unemploy- 
ment. A new equilibrium will result in which the originally unemployed permanently receive both wages and a substantial rental income. (The full story is complicated because nutrition (i.e., body weight) cannot be instantaneously redistributed. First the government could control the economy for a finite number of periods in order to position each dynasty's nutrition at or above $\tilde{n}$ (Lemma 12 in Appendix). Then the government could endow each dynasty with land $K>\underline{K}$ and relinquish control. The ensuing nonstationary equilibrium would exhibit full employment in every period and stationary prices; consumption, and land ownership. Each dynasty's nutrition would converge to $(r K+q-\alpha)(1-\beta)^{-1}$.)

We expect that somewhere in the unit interval there are threshold land reforms $\theta$ and $\bar{\theta}$ such that if $\theta>\bar{\theta}$, everyone will eventually be employed, and if $\theta<\underline{\theta}$, the originally unemployed will revert back to perpetual unemployment. But the story is complicated. Nonstationary prices will result if $\theta$ is large enough to make the originally unemployed want to accumulate land for future employment. Moreover, if $\theta \in[\underline{\theta}, \bar{\theta}]$, the originally unemployed may split into two classes enjoying equal utility in period 1: one accumulating land and the other decumulating.

Second, assume endogenous survival. Once again, small land reforms ( $\theta$ near zero) would not have any effect on unemployment (Stability Theorem 1 ). But, in contrast to the previous case, the new land distribution would not result in a stationary equilibrium. Rather, unemployed dynasties would seek to decumulate land because of their low survival probabilities. Since the unemployed want to sell land, the employed must be enticed to buy it. Yet the employed wish constant consumption streams because of the concavity of $G$. As a result, the price of land would fall immediately following the land reform, and then it would climb back to its original level. Bigger land reforms under endogenous survival are very similar to those discussed previously under exogenous survival, except for the fact that variable discount factors will further complicate the nonstationary dynamic 
equilibria that must be studied in order to calculate the threshold land reforms $\underline{\theta}$ and $\bar{\theta}$.

The dynamic stability of general equilibrium paths has been studied under convex structures by Bewley (1982), Coles (1983), Lucas and Stokey (1984), Yano (1984), Epstein (1987), and Benhabib, Jafarey, and Nishimura (1988). However, the pervasive nonconvexities of our model preclude an application of their techniques. The formulation of an adequate nonstationary equilibrium theory in models such as ours remains a challenging task.

APPENDIX

Lemma 1: $E$ is viable from $K$ if and only if $K / E \geq \underline{K}$.

Proof: Recall that $E$ is sald to be viable from $K$ if and only if there exist distributions $c$, $e$, and $n$ such that

$$
\begin{gathered}
\int \text { e du }=E \\
(\forall j) \quad e(j)=1 \Rightarrow n(j) \geq \tilde{n} \\
(\forall j) \quad c(j)=(1-B) n(j)+\alpha e(j) \\
\int c d \mu \leq F(K, E) .
\end{gathered}
$$

Suppose $\mathrm{E}$ is viable from $\mathrm{K}$. The four conditions defining viability imply

$$
\begin{aligned}
E \cdot((1-\beta) \tilde{n}+\alpha) & =\int_{\{j \mid e(j)=1\}}(1-\beta) \tilde{n}+\alpha d \mu \\
& \leq \int_{\{j \mid e(j)=1\}}(1-\beta) n(j)+\alpha d \mu \\
& =S_{\{j \mid e(j)=1\}} c(j) d \mu \\
& \leq S c d \mu \\
& \leq E(K, E) .
\end{aligned}
$$


By constant returns, this implies $(1-B) \tilde{n}+\alpha \leq F(K / E, 1)$, which implies $K / E$ $\geq \underline{K}$ by the definition of $\underline{K}$. On the other hand, suppose $K / E \geq \underline{K}$. Define $(c, e, n)$ by

$$
(c(j), e(j), n(j))=\left\{\begin{array}{l}
((1-\beta) \tilde{n}+\alpha, 1, \tilde{n}) \text { if } j \in[0, E] \\
(0,0,0) \text { if } j \in(E, 1] .
\end{array}\right.
$$

The first three of the four conditions defining viability are immediate. The last follows from the definition of $\underline{\underline{R}}$ and constant returns:

$$
\begin{aligned}
\int c d \mu & =E \cdot((1-\beta) \tilde{n}+\alpha) \\
& =E \cdot F(\underline{K}, 1) \\
& \leq E \cdot F(K / E, 1) \\
& =F(K, E) .
\end{aligned}
$$

Lemma 2: Suppose there is a stationary equilibrium with land $\mathrm{K}$ and employment $E$. Then $\mathrm{K} / \mathrm{E} \geq \overline{\mathrm{K}} \Rightarrow \mathrm{E}=1$.

Proof: Suppose $K / E \geq \bar{K}$. BY equilibrium property $(c)$ and the definition of $\overline{\mathrm{K}}$,

$$
\begin{aligned}
q & =F_{E}(K / E, 1) \\
& \geq F_{E}(\vec{K}, 1) \\
& =\tilde{n}+\alpha .
\end{aligned}
$$

If $E$ were strictly less than 1 , there would be an unemployed dynasty $j$ consuming $r k(j)$ in each period. But since $q \geq \tilde{n}+\alpha$, this dynasty could increase its utility by consuming $r k(j)+q$ in each period. Since this would contradict equilibrium property (a), we conclude that $E=1$. Q.E.D.

Lemma 3: Suppose $(1+r / p)^{-1}=\delta P(\tilde{n})$ and take any $\left(k_{0}, n_{0}\right)$. Then the stream defined by $(\forall t \geq 1)\left(c_{t}, e_{t}, k_{t}, n_{t}\right)=\left(r k_{0}+q, 1, k_{0}, \beta n_{t-1}+r k_{0}+q-\alpha\right)$ is the unique optimum from $\left(k_{0}, n_{0}\right)$ provided that $(\forall t \geq 1) n_{t} \geq \tilde{n}$. 
Proof: Consider the following problem: Given $\left(k_{0}, n_{0}\right)$, maximize

$$
\sum_{t=1}^{\infty}[\delta P(\tilde{n})]^{t-1} G\left(c_{t}\right)
$$

subject to $(5)-(7)$. Note that nutrition plays no role in this problem since (11) presumes exogenous survival and since the capacity constraint (4) is missing. Thus standard arguments (using $(1+r / p)^{-1}=\delta P(\tilde{n}$ ) and the strict concavity of $G$ ) imply that the unique optimizer of (11) over the feasible set $(5)-(7)$ is defined by $(\forall t \geq 1)\left(c_{t}, e_{t}, k_{t}, n_{t}\right)=\left(r k_{0}+q, 1, k_{0}\right.$, $\left.\beta n_{t-1}+r k_{0}+q-\alpha\right)$.

Since $(\forall t \geq 1) n_{t} \geq \tilde{n}$ by assumption, $\left\langle\left(c_{t}, e_{t}, k_{t}, n_{t}\right)\right\rangle_{t=1}^{\infty}$ satisfies (4). Hence $\left\langle\left(c_{t}, e_{t}, k_{t}, n_{t}\right)\right\rangle_{t=1}^{\infty}$ is the unique optimizer of (11) over the smaller feasible set $(4)-(7)$.

Note that ( 8 ) is bounded from above by (11) because $P$ is bounded from above by $P(\tilde{n})$. Also note that $(8)$ and (11) assume the same value at $\left\langle\left(c_{t}, e_{t}, k_{t}, n_{t}\right)\right\rangle_{t=1}^{\infty}$. Thus $\left\langle\left(c_{t}, e_{t}, k_{t}, n_{t}\right)\right\rangle_{t=1}^{\infty}$ is the unique optimizer of $(8)$ over the feasible set $(4)-(7)$. Q.E.D.

\section{Proof for the Characterization Theorem: Suppose there is an equili-} brium with land $K$ and employment $E$. Since any equilibrium $E$ must be viable from $\mathrm{X}$, Lemma 1 implies (9). Lemma 2 implies (10).

on the other hand, suppose that $(K, E)$ satisfies (9) and (10). Define prices by

$$
\begin{gathered}
q=F_{E}(K, E), \\
r=F_{K}(K, E), \text { and } \\
p=\delta P(\tilde{n})(1-\delta P(\tilde{n}))^{-1} r .
\end{gathered}
$$

Since $X>0$ by assumption, since $E>0$ by $(10)$, and since $F$ is strictly increasing over $\mathbb{R}_{++^{\prime}}^{2}$, we have $(q, r, p)>(0,0,0)$. Define the distributions $(c, e, k, n)$ by 


$$
\begin{aligned}
& (c(j), e(j), k(j), n(j))= \\
& \left\{\begin{array}{l}
\left(r K / E+q, 1, K / E,(r K / E+q-\alpha)(1-\beta)^{-1}\right) \text { if } j \in[0, E] \\
(0,0,0,0) \text { if } j \in(E, 1] .
\end{array}\right.
\end{aligned}
$$

Properties $(b)-(c)$ follow immediately from the definitions of $k$, $q$, and $r$. Property (d) follows from the fact that $(\forall j \in[0, E]) c(j)=F(K, E) / E$ by Euler's Theorem. Property (a) is more involved. First take $f \in[O, E]$. Euler's Theorem, (9), and the definition of K imply

$$
\begin{aligned}
n(j) & =(r K / E+q-\alpha)(1-\beta)^{-1} \\
& =(F(K / E, 1)-\alpha)(1-\beta)^{-1} \\
& \geq(E(K, 1)-\alpha)(1-\beta)^{-1} \\
& =\tilde{n} .
\end{aligned}
$$

Hence the stationary $\operatorname{stream}(c(j), e(j), k(j), n(j))$ is optimal by Lemma 3 . Second take $f \in(E, 1)$. Since this case is vacuous when $E=1$, we may assume $E<1$. Thus, by $(10)$ and the definition of $\overline{\mathrm{K}}$,

$$
\begin{aligned}
q & =F_{E}(K / E, 1) \\
& <F_{E}(\bar{K}, 1) \\
& =\tilde{n}+\alpha .
\end{aligned}
$$

Therefore, since $n(j)=0$, it is impossible to work without first accumulating land or nutrition. But since $k(j)=0$, it is also impossible to accumulate land or nutrition without labor income. Therefore the stationary stream $(c(j), e(j), k(j), n(j))=(0,0,0,0)$ is the only feasible stream. (Section 5.1 makes the distinct and significant observation that this optimun is locally stable.) Q.E.D.

Lemma 4: $(1+r / p)^{-1}=\delta P(\tilde{n})$ in any stationary equilibrium such that ( $\exists j)(k(j), n(j)) \in \bar{s} \sim(\bar{k}, \bar{n})$. 
Proof: Suppose $\delta P(\tilde{\Omega})>(1+r / p)^{-1}$. By assumption, there is a dynasty whose stationary optimum $(k, n)$ obeys $(k, n) \in S \sim(\bar{k}, \bar{n})$. Since $n>\tilde{n}$ by the definition of $(\bar{k}, \bar{n})$, it is feasible to increase first-period savings while maintaining first-period nutrition. Standard arguments using $\delta P(\tilde{n})>$ $(1+r / p)^{-1}$ then show that this accumulation of land enables a consumption stream which yields higher utility than the original stationary stream.

Suppose $\delta P(\tilde{n})<(1+r / P)^{-1}$. By assumption, there is a dynasty whose stationary optimum $(k, n)$ obeys $(k, n) \in S \sim(\bar{k}, \bar{n})$. Note $k>0$ by the definition of $(\bar{k}, \bar{n})$. Let $s \geq 1$ satisfy both

$$
\begin{gathered}
{\left[\delta P(\tilde{n}) /(1+r / p)^{-1}\right]^{s}<k(1+r / p) G^{\prime}(r k+q)(2 u)^{-1} \text { and }} \\
{\left[G\left(r k+q+(1+r / p)^{-s+1} k\right)-G(r k+q)\right] /\left[(1+r / p)^{-s+1} k\right]>G^{\prime}(r k+q) / 2,}
\end{gathered}
$$

where $u=G(r k+q)(1-\delta P(\tilde{n}))^{-1}$. Such an $s$ must exist because $k>0$, because $\delta P(\tilde{R})<(1+r / P)^{-1}$, and because $G^{\prime}$ is always positive. Consider taking the land $k$ in period $s$ and consuming 1 ts discounted value $(1+r / p)^{-s+1} k$ in the period 1. This decumulation would yield a utility gain in period 1 of $G\left(r k+q+(1+r / p)^{-s+1} k\right)-G(r k+q)$. The loss, evaluated at period $s+1$, is at most $u=G(r k+q)(1-\delta P(\tilde{n}))^{-1}$. This upper bound recognizes that by stripping away all land in period $s$, we may sacrifice all employment after period s. Thus the loss, evaluated at period 1 , is at most $[\delta P(\tilde{\mathbf{n}})]^{\mathbf{s}} \mathrm{u}$. The gain exceeds the loss:

$$
\begin{aligned}
& G\left(r k+q+(1+r / p)^{-s+1} k\right)-G(r k+q) \\
> & {\left[G^{\prime}(r k+q) / 2\right]\left[(1+r / p)^{-s+1} k\right] } \\
> & {\left[\delta P(\tilde{n}) /(1+r / p)^{-1}\right]^{s} k^{-1}(1+r / p)^{-1} u\left[(1+r / p)^{-s+1} k\right] } \\
= & {[\delta P(\tilde{n})]^{s} u . }
\end{aligned}
$$

(the two inequalities follow from the inequalities defining s). Thus we conclude that this decumulation yields a utility higher than that of the original stationary stream.

Q.E.D. 
Lemma 5: Suppose $(1+r / P)^{-1}=\delta P(\tilde{\mathrm{n}})$. Then an optimum exists from any $\left(k_{0}, n_{0}\right)$

Proof: Take any $\left(k_{0}, n_{0}\right) \in \mathbb{R}_{+}^{2}$. Following streufert $((1989 b)$, Section 6). Let $A=\mathbb{R}_{+} \times(0,1) \times \mathbb{R}_{+}^{2}$ be the action space, let $a_{0}=\left(0,0, k_{0}, n_{0}\right)$ be the initial action, and let $z_{t}=\left(c_{t}, e_{t}, k_{t}, n_{t}\right)$ be action in period $t$. Define the nonnegative aggregator $\mathrm{W}: A \times \mathbb{R}_{+} \rightarrow \mathbb{R}_{+}$by

$$
W\left(a_{t}, u_{t+1}\right)=G\left(c_{t}\right)+\delta P\left(n_{t}\right) u_{t+1} \cdot
$$

By assumption, $G$ and $S$ are continuous, $\delta>0$, and $\left(\forall n_{t}\right) P\left(n_{t}\right)>0$. Thus $W$ is continuous, and it is strictly increasing in future utility. Define the production correspondence $T: A \Rightarrow A$ by

$$
\begin{aligned}
T\left(a_{t-1}\right) & =\left\{a_{t} \mid e_{t}=0 \text { and }\left(k_{t}, n_{t}\right)=f^{0}\left(k_{t-1}, n_{t-1}, c_{t}\right)\right\} \\
& \cup\left(a_{t} \mid e_{t}=1, n_{t} \geq \tilde{n} \text {, and }\left(k_{t}, n_{t}\right)=f^{1}\left(k_{t-1}, n_{t-1}, c_{t}\right)\right\},
\end{aligned}
$$

where $f^{0}: R_{+}^{3} \rightarrow \mathbb{R}_{+}^{2}$ is defined by

$$
\begin{aligned}
& k_{t}=k_{t-1}+\left(r k_{t-1}-c_{t}\right) / p \\
& n_{t}=B n_{t-1}+c_{t^{\prime}}
\end{aligned}
$$

and $f^{1}: \mathbb{R}_{+}^{3} \rightarrow \mathbb{R}_{+}^{2}$ is defined by

$$
\begin{aligned}
& k_{t}=k_{t-1}+\left(r k_{t-1}+q-c_{t}\right) / p \\
& n_{t}=B n_{t-1}+c_{t}-\alpha .
\end{aligned}
$$

To see that $T$ is u.s.c., take any $a_{t-1} \in A$. Define $K \subseteq A$ by $K=\left[0, c_{t-1}+1\right]$ $\times\{0,1\} \times\left[0, k_{t-1}+1\right] \times\left[0, n_{t-1}+1\right]$. Note that the restriction of $T$ to $k$ maps $K$ into $\left[0,(p+r) k_{t-1}+q\right] \times\{0,1\} \times\left[0,(1+r / p) k_{t-1}+q / p\right] \times\left[0, B n_{t-1}+(p+r) k_{t-1}+q\right]$. Also note that the restriction of $T$ to $K$ is closed (as defined by Berge (1963), p. 111) because $f^{0}$ and $f^{1}$ are continuous. Thus the restriction of $T$ to $k$ is u.s.c. (Berge, p. 112, Corollary to Theorem 7 ). Since $a_{t-1}$ is in the 
interior of $K$, this implies that $T$ is compact-valued and u.s.c. at a ${ }_{t-1}$ (as defined on Berge, p. 109). Because this holds at all $a_{t-1} \in A, T$ is u.s.c. Since $a_{t} \in T\left(a_{t-1}\right)$ implies $k_{t} \leq(1+r / p) k_{t-1}+q / p, a_{t} \in T^{t}\left(a_{0}\right)$ implies

$$
\begin{aligned}
k_{t} & \leq(1+r / p)^{t} k_{0}+(q / p) \Sigma_{z=0}^{t-1}(1+r / p)^{z} \\
& \leq(1+r / p)^{t} k_{0}+(q / p) \int_{z=0}^{t}(1+r / p)^{z} \\
& \leq(1+r / p)^{t} k_{0}+(q / p)(1+r / p)^{t} / 1 n(1+r / p) \\
& =(1+r / p)^{t}\left(k_{0}+q\right),
\end{aligned}
$$

where $\phi=(q / p) / \ln (1+r / p)>0$. Thus, $a_{t} \in T^{t}\left(a_{0}\right)$ implies

$$
\begin{aligned}
c_{t} & \leq(p+r) k_{t-1}+q \\
& \leq(p+r)(1+r / p)^{t-1}\left(k_{0}+\phi\right)+q \\
& =p(1+r / p)^{t}\left(k_{0}+\phi\right)+q .
\end{aligned}
$$

Also note that by assumption, there are $\gamma<1$ and $\bar{c} \in \mathbb{R}+$ such that $(\forall c \geq \bar{c})$ $G(c) \leq c^{\gamma}$. Hence

$$
\begin{aligned}
(\forall t \geq 1) & \sup w\left(T^{t}\left(a_{0}\right), 0\right) \\
\leq & G\left(p(1+r / p)^{t}\left(k_{0}+\phi\right)+q\right) \\
\leq & G(\bar{c})+\left(p(1+r / p)^{t}\left(k_{0}+\phi\right)+q\right)^{\gamma} \\
\leq & p^{\gamma}\left(k_{0}+\phi\right)^{\gamma}\left[(1+r / p)^{\gamma}\right]^{t}+\left[G(\bar{c})+q^{\gamma}\right] .
\end{aligned}
$$

Hence $b=(1+r / p)^{\gamma}$ bounds the growth of $\left\langle\sup W\left(T^{t}\left(a_{0}\right), 0\right)\right\rangle_{t=1}^{\infty}$ (as defined in Streufert $(1989 b)$, Section 6.2$)$. Time perspective is bounded by $d^{*}=\bar{d}=$ $\delta P(\tilde{\mathrm{n}})<1$. Because $(1+r / P)^{-1}=\delta P(\tilde{\mathrm{n}})$ by assumption,

$$
\begin{aligned}
d^{* b} & =\delta P(\tilde{n})(1+r / p)^{\gamma} \\
& <\delta P(\tilde{n})(1+r / p) \\
& =\delta P(\tilde{n})(\delta P(\tilde{n}))^{-1} \\
& =1 .
\end{aligned}
$$

An action stream satisfies $(4)-(7)$ if and only if $(\forall t \geq 1) a_{t} \in T\left(a_{t-1}\right)$, and 
the objective function $(8)$ is the zero-limit of $W$ (as defined in streufert $(1989 b)$, section 6.1). Thus biconvergence follows from streufert ((1989b), Result 5), and the existence of an optimum follows from streufert ((1989a), Theorem C).

Lemma $6:$ Suppose $(1+r / p)^{-1}=\delta P(\tilde{n})$. Then if $\left(k_{0}, n_{0}\right)>(\bar{k}, \bar{n})$, the unique optimum is defined by $(\forall t \geq 1)\left(c_{t}, e_{t}, k_{t}, n_{t}\right)=\left(r k_{0}+q, 1, k_{0}\right.$. $\left.\beta n_{t-1}+r k_{0}+q-\alpha\right)$. (Note $\left.l i m_{t \rightarrow \infty}\left(k_{t}, n_{t}\right)=\left(k_{0},\left(r k_{0}+q-\alpha\right)(1-\beta)^{-1}\right) \in \bar{s}.\right)$

Proof: Consider the stream $\left\langle\left(c_{t}, e_{t}, k_{t}, n_{t}\right)\right\rangle_{t=1}^{\infty}$ defined by $(\forall t \geq 1)$ $\left(c_{t}, e_{t}, k_{t}, n_{t}\right)=\left(r k_{0}+q, 1, k_{0}, B n_{t-1}+r k_{0}+q-\alpha\right)$. Since $r k_{0}+q-\alpha$ is constant, $\left\langle n_{t}\right\rangle_{t=1}^{\infty}$ converges monotonically to $\left.\left(r k_{0}+q-\alpha\right)(1-\beta)^{-1}\right\rangle$ $(r \bar{k}+q-\alpha)(1-\beta)^{-1} \geq \tilde{n}$ (the weak inequality follows from the definition of $(\bar{k}, \bar{n}))$. Thus, since $n_{0}>\bar{n} \geq \tilde{n}$, we have $(\forall t \geq 1) n_{t} \geq \tilde{n}$. Since $(1+r / p)^{-1}=$ $\delta P(\tilde{\Omega})$ by assumption, we may apply Lemma 3 .

Q.E.D.

Lemma 7 : Assume $K<\bar{K}$. Take any stationary equilibrium prices. Then there exists $a(\underline{\underline{k}}, \underline{\underline{n}}) \in \underline{\underline{S}}(0,0)$ and $a \hat{n}<\tilde{n}$ with the following property: If $\left(k_{0}, n_{0}\right)<(\underline{\underline{k}}, \underline{n})$, if $\left\langle\left(c_{t}, e_{t}, k_{t}, n_{t}\right)\right\rangle_{t=1}^{\infty}$ is feasible from $\left(k_{0}, n_{0}\right)$, and if $(\exists t \geq 1) n_{t}>\hat{n}$, then there is a time $s \geq 1$ such that $k_{s}>k \cdot(\forall t \leq s-1)$ $k_{t} \leq \underline{\underline{k}},(\forall t \leq s) e_{t}=0$, and $(\forall t \leq s) n_{t} \leq \hat{n}$. (Intuitively, when $k<\bar{k}$, there is $a(\underline{\underline{k}}, \underline{\underline{n}})>(0,0)$ such that if you start out below (k, accumulate at least $\underline{\underline{k}}$ units of land before working. During this accumulation period, your nutrition cannot exceed $\hat{n}<\tilde{n}$.)

Proof: We have $q<\tilde{n}+\alpha$ by $K / E \leq K<\bar{K}$ and the definition of $\bar{K}$. Thus we may choose $(\underline{\underline{k}}, \underline{\underline{n}}) \in \underline{\underline{S}}(0,0)$ to satisfy $\beta \underline{\underline{\underline{n}}}+(p+r) \underline{\underline{k}}+q-\alpha<\tilde{n}$. Define $\hat{\mathrm{n}}=\beta \underline{\underline{n}}+(p+r) \underline{\underline{k}}+q-\alpha$.

First consider any $\left(k_{t-1}, n_{t-1}\right)$ such that $k_{t-1} \leq k$ and $p k_{t-1}+n_{t-1}=$ $p \underline{\underline{k}}+\underline{\underline{g}}$. If $e_{t}$ were equal to $1,(5)-(7)$ would imply 


$$
\begin{aligned}
& n_{t}=B n_{t-1}+c c_{t}-\alpha \\
& \leq B n_{t-1}+(p+r) k_{t-1}+q-\alpha
\end{aligned}
$$

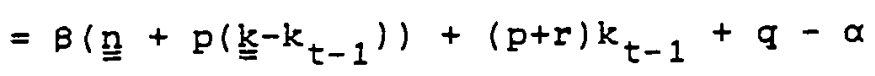

$$
\begin{aligned}
& =\beta \underline{\underline{n}}+(p+r) \underline{\underline{k}}+q-\alpha-(r+(1-\beta) p)\left(\underline{\underline{k}-k_{t-1}}\right) \\
& \leq \beta \underline{\underline{n}}+(p+r) \underline{\underline{k}}+q-\alpha \\
& =\hat{n} \text {. }
\end{aligned}
$$

Thus, $\hat{n}<\tilde{n}$ and $(4)$ imply $e_{t}=0$. Since $e_{t}=0$, we may combine $(5)$ and $(6)$ to eliminate $c_{t}$ and obtain

$$
p k_{t}+n_{t}=p k_{t-1}+n_{t-1}+\left[r k_{t-1}-(1-\beta) n_{t-1}\right]
$$

Since $\underline{\underline{n}}=r \underline{\underline{k}}(1-\beta)^{-1}$ by $(\underline{\underline{k}}, \underline{\underline{n}}) \in \underline{S}$.

$$
\begin{aligned}
& r k_{t-1}-(1-\beta) n_{t-1} \\
= & r k_{t-1}-(1-\beta)\left(\underline{\underline{n}}+p\left(\underline{\underline{k}}-k_{t-1}\right)\right) \\
= & r \underline{\underline{k}}-(1-\beta) \underline{\underline{n}}-(p(1-\beta)+r)\left(\underline{\left.\underline{k}-k_{t-1}\right)}\right. \\
\leq & r \underline{\underline{k}}-(1-\beta) \underline{\underline{n}} \\
= & 0 .
\end{aligned}
$$

The previous two equations imply $p k_{t}+n_{t} \leq p k_{t-1}+n_{t-1}=p \underline{\underline{k}}+\underline{\underline{n}}$.

Now take any $\left(k_{t-1}, n_{t-1}\right)$ such that $k_{t-1} \leq \underline{\underline{k}}$ and $p k_{t-1}+n_{t-1} \leq p \underline{\underline{k}}+\underline{\underline{n}}$.

Since there is some $n_{t-1}^{+} \geq n_{t-1}$ such that $p k_{t-1}+n_{t-1}^{+}=p k+n$, the previous paragraph implies that if $(4)-(7)$ are satisfied, then $n_{t} \leq \hat{n}$, $e_{t}=0$, and $p k_{t}+n_{t} \leq p \underline{\underline{k}}+\underline{\underline{n}}$.

Finally, let $\left(k_{0}, n_{0}\right) \ll(\underline{\underline{k}}, \underline{\underline{n}})$, let $\left\langle\left(c_{t}, e_{t}, k_{t}, n_{t}\right)\right\rangle_{t=1}^{\infty}$ be feasible from $\left(k_{0}, n_{0}\right)$, and suppose $(\exists t \geq 1) n_{t}>\hat{n}$. Define

$$
\left.s=\min \left\{t \geq 0 \mid k_{t}>\underline{\underline{k}} \text { or } p k_{t}+n_{t}\right\rangle p \underline{\underline{k}}+\underline{\underline{n}}\right\}
$$

This set is nonempty because $n_{t}>\hat{n}$ implies $k_{t-1}>\underline{k}$ or pk $k_{t-1}+n_{t-1}>p k+$ $\underline{n}$ by the previous paragraph. Since $\left(k_{0}, n_{0}\right)<(\underline{\underline{k}}, \underline{\underline{n}})$, we have $s \geq 1$. 
The definition of $s$ assures $(\forall t \leq s-1) k_{t} \leq k$. Note $\left(k_{s-1} \cdot n_{s-1}\right)$ is well-defined because $s \geq 1$. Since $\left(k_{s-1}, n_{s-1}\right)$ satisfies $k_{s-1} \leq \underline{k}$ and pk $k_{s-1}$ $+n_{s-1} \leq p \underline{\underline{k}}+\underline{\underline{n}}$ by the definition of $s$, the paragraph before the previous one implies $\mathrm{pk}_{s}+\mathrm{n}_{s} \leq \mathrm{pk}+\underline{\underline{n}}$. Thus the definition of $s$ implies $k_{s}>\underline{k}$. Finally, since $(\forall t \leq s-1) k_{t} \leq k$ and $p k_{t}+n_{t} \leq p k+\underline{\underline{k}}$ by the definition of $s$, the paragraph before the previous one implies that $(\forall t \leq s) e_{t}=0$ and $(\forall t \leq s) n_{t} \leq \hat{n}$.

Q.E.D.

Lemma 8: Assume $\mathrm{K}<\overline{\mathrm{K}}$ and endogenous survival. Take any stationary equilibrium prices such that $(1+r / p)^{-1}=\delta P(\tilde{n})$. Then there is $a(\underline{k}, \underline{n}) \in$ $\underline{S}(0,0)$ such that if $\left(k_{0}, n_{0}\right) \ll(\underline{k}, \underline{n})$, then every optimum is such that $(\forall t \geq 1) e_{t}=0,(\forall t \geq 1) k_{t} \leq k_{t-1}$, and $\lim m_{t \rightarrow \infty} c_{t}=\lim \mathrm{l}_{t \rightarrow \infty} k_{t}=\lim \mathrm{m}_{t \rightarrow \infty} n_{t}=0$.

Proof: Define $(\underline{\underline{k}}, \underline{n}) \in \underline{\mathrm{s}} \sim(0,0)$ and $\tilde{\mathrm{n}}<\tilde{\mathrm{n}}$ via Lemma 7 . Note $(1+r / p)^{-1}$ $=\delta P(\tilde{n})$ by assumption. Let d denote this equilibrium discount factor, let $\hat{d}=\delta P(\hat{n})<d$, and let $a=\ln \hat{d} / \ln d$. Note that $\hat{d}=d^{a}$, and that $a>1$ since $\ln \hat{d}<\ln d<0$. Define $\underline{k} \in(0, \underline{\underline{k}})$ so that

$$
G^{\prime}((p+r) \underline{k})>d u \underline{k}^{-a} \underline{k}^{a-1}
$$

where $u=G\left(r d^{-1} \underline{\underline{k}}+q\right)(1-d)^{-1}$. Such a $\underline{k}$ exists because $1 i m_{k \rightarrow 0} G^{\prime}((p+r) k)>$ O since $G$ is strictly concave, and because $l_{i m_{k \rightarrow 0}} d_{u k}{ }^{-a_{k}}{ }^{a-1}=0$ since $a>1$. Define $\underline{n}$ to be the second coordinate of the point on $\underline{S}$ whose first coordinate is $\underline{k}$. Since $0<\underline{k}<\underline{\underline{k}}, 0<\underline{\underline{n}}<\underline{\underline{n}}$.

Take any $\left(k_{0}, n_{0}\right) \ll(\underline{k}, \underline{n})$. Let $\left\langle\left(c_{t}, e_{t}, k_{t}, n_{t}\right)\right\rangle_{t=1}^{\infty}$ be a feasible stream from $\left(k_{0}, n_{0}\right)$ such that $(\exists t \geq 1) n_{t} \geq \hat{n}$. By Lemma 7 , there is an $s \geq 1$ such that $k_{s}>\underline{\underline{k}},(\forall t \leq s-1) k_{t} \leq \underline{\underline{k}},(\forall t \leq s) e_{t}=0$, and ( $\left.\forall t \leq s\right) n_{t} \leq \hat{n}$. Consider taking the land $k_{s}$ and consuming in the first period its present discounted value $d^{s-1} k_{s}$. This would yield a utility gain in the first period of $G\left(c_{1}+d^{s-1} k_{s}\right)-G\left(c_{1}\right)$. Since $e_{s}=0$ and $k_{s-1} \leq k$, we have that $k_{s} \leq d^{-1} k_{s-1} \leq d^{-1} k$. Thus, the utility loss, evaluated at period $s+1$, is 
at most $u=G\left(r d^{-1} \underline{\underline{k}}+q\right)(1-d)^{-1}$. This upper bound recognizes that by stripping away $k_{s}$ we may sacrifice employment in every period after period s. Since $(\forall t \leq s) n_{t} \leq \hat{n}$, the utility loss evaluated at period 1 is at most $\hat{d}^{s} u$. The gain exceeds the loss:

$$
\begin{aligned}
& G\left(c_{1}+d^{s-1} k_{s}\right)-G\left(c_{1}\right) \\
> & G^{\prime}((p+r) \underline{k}) d^{s-1} k_{s} \\
> & (\underline{k} / \underline{k})^{a}\left(d^{s} k_{s} / \underline{k}\right) u \\
> & (\underline{k} / \underline{k})^{a}\left(d^{s} k_{s} / \underline{k}\right)^{a} u \\
> & a^{a s} u \\
= & \hat{d}^{s} u .
\end{aligned}
$$

The first inequality follows from the strict concavity of $G, c_{1} \leq(p+r) k_{0}$ (since $e_{1}=0$ ), and $k_{0}<\underline{k}$. The second holds by the definition of $\underline{k}$. The third follows from $k_{s} \leq d^{-s_{k}} k_{0}$ (since $(\forall t \leq s) e_{t}=0$ ), $k_{0}<\underline{k}$, and a $>1$. The fourth holds because $k_{s}>\underline{k}$. Since the gain exceeds the loss, we conclude that every optimum from $\left(k_{0}, n_{0}\right)$ satisfies $(\forall t \geq 1) n_{t} \leq \hat{n}$.

Again, take any $\left(k_{0}, n_{0}\right) \ll(\underline{k}, \underline{n})$. Let $\left\langle\left(c_{t}, e_{t}, k_{t}, n_{t}\right)\right\rangle_{t=1}^{\infty}$ be an optimum from $\left(k_{0}, n_{0}\right)$. By the previous paragraph, $(\forall t \geq 1) n_{t} \leq \hat{n}$. Note that $(\forall t \geq 1) n_{t} \leq \hat{n}$ implies $(\forall t \geq 1) e_{t}=0$ by $(4)$, and thus $\left\langle\left(c_{t}, e_{t}, k_{t}, n_{t}\right)\right\rangle_{t=1}^{\infty}$ optimizes ( 8 ) over the smaller feasible set defined by $(5)-(7)$ and $(\forall t \geq 1)$ $e_{t}=0$. Also note $(\forall t \geq 1) n_{t} \leq \hat{n}$ implies $(\forall t \geq 1) \delta P\left(n_{t}\right) \leq \hat{d}<d$. Therefore, standard arguments demonstrate that $\left\langle k_{t}\right\rangle_{t=1}^{\infty}$ is monotonically decreasing and that $\lim _{t \rightarrow \infty} k_{t}=\lim _{t \rightarrow \infty} c_{t}=\lim _{t \rightarrow \infty} n_{t}=0$.

Q.E.D.

Lemma 9: Assume $\mathrm{K}<\overline{\mathrm{K}}$, exogenous survival, and $\lim _{c \rightarrow 0^{\prime}}(c)=+\infty$.

Take any stationary equilibrium prices such that $(1+r / p)^{-1}=\delta P(\bar{n})$. Then there is a $(\underline{k}, \underline{n}) \in \underline{S} \sim(0,0)$ such that if $\left(n_{0}, k_{0}\right) \ll(\underline{k}, \underline{n})$, the unique optimum is defined by $(\forall t \geq 1)\left(c_{t}, e_{t}, k_{t}, n_{t}\right)=\left(r k_{0}, 0, k_{0}, B n_{t-1}+r k_{0}\right)$. (Note $\left.\lim _{t \rightarrow \infty}\left(k_{t}, n_{t}\right)=\left(k_{0}, r k_{0}(1-\beta)^{-1}\right) \in \underline{S}_{\cdot}\right)$ 
Proof: Define $(\underline{\underline{k}}, \underline{\underline{n}}) \in \underline{S} \sim(0,0)$ and $\hat{n}<\tilde{n}$ via Lemma 7 . Note $(1+r / p)^{-1}$ $=\delta P(\tilde{n})$ by assumption. Let d denote this equilibrium discount factor. Define $\underline{k} \in(0, \underline{\underline{k}})$ so that

$$
G^{\prime}((p+r) \underline{k})>\operatorname{duk}^{-1}
$$

where $u=G\left(r d^{-1} \underline{\underline{k}}+q\right)(1-d)^{-1}$. Such a $\underline{k}$ exists because $\lim _{k \rightarrow 0} G^{\prime}((p+r) k)=$ $+\infty$. Define $\underline{n}$ to be the second coordinate of the point on $\underline{S}$ whose first coordinate is $\underline{k}$. Since $0<\underline{k}<\underline{\underline{k}}, 0<\underline{n}<\underline{\underline{n}}$.

Take any $\left(k_{0}, n_{0}\right) \ll(\underline{k}, \underline{n})$. Let $\left\langle\left(c_{t}, e_{t}, k_{t}, n_{t}\right)\right\rangle_{t=1}^{\infty}$ be a feasibie stream from $\left(k_{0}, n_{0}\right)$ such that $(\exists t \geq 1) n_{t}>\hat{n}$. By Lemma $z$, there is an $s \geq 1$ such that $k_{s}>\underline{\underline{k}},(\forall t \leq s-1) k_{t} \leq k,(\forall t \leq s) e_{t}=0$, and $(\forall t \leq s) n_{t} \leq \hat{n}$. Consider taking the land $k_{s}$ and consuming in the first period its present discounted value $d^{s-1} k_{s}$. This would yield a utility gain in the first period of $G\left(c_{1}+d^{s-1} k_{s}\right)-G\left(c_{1}\right)$. Since $e_{s}=0$ and $k_{s-1} \leq k$, we have that $k_{s} \leq d^{-1} k_{s-1} \leq d^{-1} \underline{k}$. Thus, the utility loss is at most $d^{s} u=d^{s} G\left(\mathrm{rd}^{-1} \underline{k}\right.$ $+q)(1-d)^{-1}$. This upper bound recognizes that by stripping away $k_{s}$ we may sacrifice employment in every period after period $s$. The gain exceeds the loss:

$$
\begin{aligned}
& G\left(c_{1}+d^{s-1_{k}}\right)-G\left(c_{1}\right) \\
> & G^{\prime}((p+r) \underline{k}) d^{s-1} k_{s} \\
> & \left(k_{s} / \underline{k}\right) d^{s} u \\
> & d^{s} u .
\end{aligned}
$$

The first inequality follows from the strict concavity of $G, c_{1} \leq(p+r) k_{0}$ (since $e_{1}=0$ ), and $k_{0}<\underline{k}$. The second holds by the definition of $\underline{k}$. The third follows from $k_{s}>k$. Since the gain exceeds the loss, we conclude that every optimum from $\left(k_{0}, n_{0}\right)$ satisfies $(\forall t \geq 1) n_{t} \leq \bar{n}$.

Again, take any $\left(k_{0}, n_{0}\right) \ll(\underline{k}, \underline{n})$. Let $\left\langle\left(c_{t}, e_{t}, k_{t}, n_{t}\right)\right\rangle_{t=1}^{\infty}$ be an optimum from $\left(k_{0}, n_{0}\right)$. By the previous paragraph, $(\forall t \geq 1) n_{t} \leq \hat{n}$. Note that 
$(\forall t \geq 1) n_{t} \leq \hat{n}$ implies $(\forall t \geq 1) e_{t}=0$ by $(4)$, and thus $\left\langle\left(c_{t}, e_{t}, k_{t}, n_{t}\right)\right\rangle_{t=1}^{\infty}$ optimizes ( 8 ) over the smaller feasible set defined by $(5)-(7)$ and $(\forall t \geq 1)$ $e_{t}=0$. Therefore, exogenous survival and the strict concavity of $G$ imply that $(\forall t \geq 1) c_{t}=r k_{0}$ and $(\forall t \geq 1) k_{t}=k_{0}$. Thus $(\forall t \geq 1) n_{t}=\beta n_{t-1}+r k_{0}$. Since these facts are true for all optima, and since an optimum exists by Lemma 5 , the optimum is unique.

Lemma 10: Suppose $(1+r / p)^{-1}=\delta P(\tilde{n})$ and $\bar{k}>0$. Then there is an $\varepsilon>0$ such that if $k_{0} \in(\bar{k}-\varepsilon, \bar{k})$, then every optimum from $\left(k_{0}, \tilde{n}\right)$ is such that $k_{1}<k_{0}$.

Proof: Note $(1+r / p)^{-1}=\delta P(\tilde{n})$ by assumption. Let $d$ be this equlibrium discount factor. Define $s \geq 1$ such that

$$
\sum_{t=1}^{s} d^{t-1} G(r \bar{k}+q)>G(r \bar{k}+d q)(1-d)^{-1}
$$

Such an $s$ exists because $\Sigma_{t=1}^{\infty} d^{t-1} G(r \bar{k}+q)=G(r \bar{k}+q)(1-d)^{-1}$. Define $\varepsilon \epsilon$ $\left(0 ; d^{s} \bar{k}\right) . \quad F i x k_{0} \in(\bar{k}-\varepsilon, \bar{k})$.

Let $\left\langle\left(c_{t}, e_{t}, k_{t}, n_{t}\right)\right\rangle_{t=1}^{\infty}$ be a feasible stream from $\left(k_{0}, \tilde{n}\right)$ that satisfies $k_{1} \geq k_{0}$. If $e_{1}$ were equal to 1 , then $(5),(6)$, the definition of $\bar{k}$, and the assumption $\bar{k}>0$ would imply

$$
\begin{aligned}
\mathrm{n}_{1} & =\tilde{n}-p\left(k_{1}-k_{0}\right)+\left[r k_{0}+q-(1-\beta) \tilde{n}-\alpha\right] \\
& <\tilde{n}-p\left(k_{1}-k_{0}\right)+[r \bar{k}+q-(1-\beta) \tilde{n}-\alpha] \\
& =\tilde{n}-p\left(k_{1}-k_{0}\right) \\
& \leq \tilde{n} .
\end{aligned}
$$

Thus (4) implies $e_{1}=0$. Therefore, total income discounted to period 1 is bounded by $(r \bar{k}+q)(1-d)^{-1}-q=(r \bar{k}+d q)(1-d)^{-1}$. Thus utility is bounded by $G(r \bar{k}+d q)(1-d)^{-1}$.

Now we relax $k_{1} \geq k_{0}$ and construct a feasible stream that exceeds this upper bound. First consider all $t \leq s$. Define $\left(c_{t}, e_{t}, k_{t}, n_{t}\right)=(r \bar{k}+q, 1$, 
$\left.\bar{k}-d^{-t}\left(\bar{k}-k_{0}\right), \tilde{n}\right)$. (4) obviously holds. (5) holds since $r \bar{k}+q=(1-\beta) \tilde{n}+$ $\alpha$ by the definition of $\bar{k}$ and by the assumption $\bar{k}>0 .(6)$ is verified by algebraic manipulation. (7) holds since $\left\langle k_{t}\right\rangle_{t=1}^{s}$ is decreasing and $\left.k_{s}\right\rangle \bar{k}-$ $\mathrm{d}^{-s} \varepsilon>0$ by the definitions of $k_{0}$ and $\varepsilon$. Second consider all $t>s$. Define $\left(c_{t}, e_{t}\right)=(0,0)$, and let $\left(k_{t}, n_{t}\right)$ evolve according to $(5)$ and $(6)$. (4) and ( $Z$ ) obviously hold. This stream yields a utility of $\Sigma_{t=1}^{s}$ $d^{t-1} G(r \vec{k}+q)$, and by the definition of $s$, this exceeds the upper bound derived when $k_{1} \geq k_{0}$.

Q.E.D.

Lemma 11: Assume $\mathrm{K} \geq \overline{\mathrm{K}}$. Take any stationary equilibrium prices such that $(1+r / p)^{-1}=\delta P(\tilde{n})$. Then for any $\left(k_{0}, n_{0}\right)$ the unique optimum is defined by $(\forall t \geq 1)\left(c_{t}, e_{t}, k_{t}, n_{t}\right)=\left(r k_{0}+q, 1, k_{0}, \beta n_{t-1}+r k_{0}+q-\alpha\right)$. (Note $\lim _{t \rightarrow \infty}$ $\left.\left(k_{t}, n_{t}\right)=\left(k_{0},\left(r k_{0}+q-\alpha\right)(1-\beta)^{-1}\right) \in \bar{s}_{.}\right)$

Proof: Consider the stream defined by $(\forall t \geq 1)\left(c_{t}, e_{t}, k_{t}, n_{t}\right)=$ $\left(r k_{0}+q, 1, k_{0} \cdot \beta n_{t-1}+r k_{0}+q-\alpha\right)$. Note that $(\forall t \geq 1) n_{t} \geq q-\alpha>\tilde{n}$ by $K / E \geq K>\bar{K}$ and the definition of $\bar{K}$. Since $(1+r / p)^{-1}=\delta P(\tilde{\Omega})$ by assumption, we may apply Lemma 3.

Q.E.D.

Lemma 12: Assume $\mathrm{K} \geq \underline{\mathrm{K}}$, and let $\mathrm{n}_{\mathrm{O}}$ be an initial nutrition distribution (not an initial stock as elsewhere in the Appendix) in which a nonzero proportion of the population is adequately nourished (i.e.. $\mu(\{j)$ $\left.\left.n_{0}(j) \geq \tilde{n},\right)>0\right)$. Then there exists a finite sequence of consumption, employment and nutrition distributions $\left\langle c_{t}, e_{t}, n_{t}\right\rangle_{t=1}^{s}$ such that (1) the equations (4), (5), and $\int c_{t} d \nu=F\left(K, \int e_{t} d \nu\right)$ are satisfied at each $t=$ $1,2, \ldots s$, and $(2)(\forall j) n_{s}(j) \geq \tilde{n}$.

Proof: Without loss of generality, assume $n_{0}$ is weakly decreasing. Define $E_{0}=\sup \left\{j \mid n_{0}(j) \geq \tilde{n}\right\}$, and define $\left\langle E_{t}\right\rangle_{t=1}^{\infty}$ recursively by

$$
E_{t}=\min \left\{1, E_{t-1}+\left[E\left(K, E_{t-1}\right)-((1-\beta) \tilde{n}+\alpha) E_{t-1}\right](\tilde{n}+\alpha)^{-1}\right\} \text {. }
$$

If $E_{t}<1$, then 


$$
\begin{aligned}
& \geq\left(1+[F(K, 1)-(1-\beta) \tilde{n}+\alpha](\tilde{n}+\alpha)^{-1}\right) \cdot E_{t-1} \\
& =\left(1+[F(K, 1)-F(\underline{K}, 1)](\tilde{n}+\alpha)^{-1}\right) \cdot E_{t-1} .
\end{aligned}
$$

Since $K>\underline{K}$ and $E_{0}>0$ by assumption, the above inequality shows that $\left\langle E_{t}\right\rangle_{t=0}^{\infty}$ is a weakly increasing sequence and that there is a finite such that $E_{S}=1$.

$$
\begin{aligned}
& \text { Define }\left\langle c_{t}, e_{t}, n_{t}\right\rangle_{t=1}^{s} \text { at each } t \text { by } \\
& \left(c_{t}(j), e_{t}(j), n_{t}(j)\right)=\left\{\begin{array}{l}
\left((1-\beta) \tilde{n}+\alpha, 1, \beta n_{t-1}(a)+(1-\beta) \tilde{n}\right) \text { if } j \in\left[0, E_{t-1}\right) \\
\left(\tilde{n}+\alpha, 1, \beta n_{t-1}(a)+\tilde{n}\right) \text { if } j \in\left[E_{t-1}, E_{t}\right] \\
\left(0,0, \beta n_{t-1}(a)\right) \text { if } j \in\left(E_{t}, 1\right] .
\end{array}\right.
\end{aligned}
$$

(4) is satisfied since $j \in\left[0, E_{t-1}\right)$ implies $n_{t-1}(a) \geq \tilde{n} . \quad$ (5) is satisfied by the definition of each $n_{t}$. Also,

$$
\begin{aligned}
\int c_{t} d \mu= & ((1-\beta) \tilde{n}+\alpha) \cdot E_{t-1}+(\tilde{n}+\alpha) \cdot\left(E_{t}-E_{t-1}\right) \\
= & ((1-\beta) \tilde{n}+\alpha) \cdot E_{t-1} \\
& +(\tilde{n}+\alpha) \cdot\left(E_{t-1}+\left[F\left(K, E_{t-1}\right)-((1-\beta) \tilde{n}+\alpha) E_{t-1}\right](\tilde{n}+\alpha)^{-1}-E_{t-1}\right) \\
= & F\left(K, E_{t-1}\right) \\
< & F\left(K, E_{t}\right) .
\end{aligned}
$$

Finally, $E_{S}=1$ and the definition of $n_{S}$ assures that $(\forall a \in[0,1]) n_{S}(a) \geq \tilde{n}$. Q.E.D. 


\section{REFERENCES}

BENHABIB, J., S. JAFAREY, AND K. NISHIMURA (1988): "The Dynamics of Efficient Intertemporal Allocations with Many Agents, Recursive Preferences, and Production", Journal of Economic Theory, 44,301-320.

BERGE, C. (1963): Topological Spaces. Edinburgh: Oliver and Boyd.

BEWLEY, T. (1982): "An Integration of Equilibrium Theory and Turnpike Theory", Journal of Mathematical Economics, 10, 233-267.

BLISS, C. and N. STERN (1978a): "Productivity, Wages and Nutrition, Part I, Theory". Journal of Development Economics, 5, 331-362.

BLISS, C. and N. STERN (1978b): "Productivity, Wages and Nutrition, Part II, Some Observations", Journal of Development Economics, 5, 363-398.

CoLEs, J. (1983): "Equllibrium Turnpike Theory with Constant Returns to Scale and Possibly Heterogeneous Discount Factors", Stanford University, IMSSS Technical Report No. 421.

DASGUPTA, P. and D. RAY (1986): "Inequality as a Determinant of Malnutrition and Unemployment, I, Basic Theory", Economic Journal, 96, 10111034 .

DASGUPTA, P. and D. RAY (1987a): "Inequality as a Determinant of Malnutrition and Unemployment, II, Policy Options", Economic Journal, 97, 177189 .

DASGUPTA, $P$, and D. RAY (1987b): "Adapting to Undernourishment: The Biological Evidence and Its Implications", forthcoming in J. Drèze and A. K. Sen (eds.). The Political Economy of Hunger. Oxford: oxford University Press.

EPSTEIN, L. (1987): "The Global Stability of Efficient Intertemporal Allocations", Econometrica, 55, 329-356.

GUPTA, M. (1987): "A Nutrition-Based Theory of Interlinkage", Burdwan University, mimeo.

GUHA, A. (1987): "Efficlency Wages and Permanent Labour", Jawaharlal Nehru University, mimeo.

KEYS, A. et al. (1950): The Biology of Human Starvation. Minneapolis: University of Minnesota Press.

LEIBENSTEIN, H. (1957): "The Theory of Underemployment in Backward Economies", Journal of Polftical Economy, 65, 91-103.

LUCAS, R. E. AND N. STOKEY (1984): "Optimal Growth with Many Consumers", Journal of Economic Theory, 32, 139-171.

MIRRLEES, J. (1975): "A Pure Theory of Underdeveloped Economies", in Agriculture in Economic Development, ed. by L. Reynolds. New Haven: Yale University Press. 
RAY, D. AND P. A. STREUfERT (1987): "On the Perpetuation of Unemployment, Undernourishment and Inequitable Land Ownership in Dynamic General Equilibrium", University of Wisconsin-Madison, SSRI Workshop Series No. 8707 .

SRINIVASAN, T. N. (1988): "Undernutrition: Concepts, Measurement and Policy Implications", Yale University, mimeo.

STIGLITZ, J. (1976): "The Efficiency Wage Hypothesis, Surplus Labour, and the Distribution of Income in LDC's", Oxford Economic Papers, 23, 185-207.

STREUFERT, P. A. (1989a): "Biconvergent Recursive Utility and Dynamic Programming: General Theory for Arbitrary Action Spaces", University of Wisconsin-Madison, SSRI Workshop Series No. 8911.

STREUFERT, P. A. (1989b): "Biconvergent Recursive Utility and Dynamic Programming: Two Special Cases using Arbitrary Action Spaces". University of Wisconsin-Madison, SSRI Workshop Series No. 8912.

YANO, M. (1984): "The Turnpike of Dynamic General Equilibrium Paths and Its Insensitivity to Initial Conditions". Journal of Mathematical Economics, 13, 235-254. 